\title{
A luminosity distribution for kilonovae based on short gamma-ray burst afterglows
}

\author{
Stefano Ascenzi ${ }^{\odot},{ }^{1,2,3,4,5 \star}$ Michael W. Coughlin, ${ }^{6}$ Tim Dietrich, ${ }^{7}$ Ryan J. Foley, \\ Enrico Ramirez-Ruiz, ${ }^{4,8}$ Silvia Piranomonte, ${ }^{1}$ Brenna Mockler, ${ }^{4,8}$ \\ Ariadna Murguia-Berthier, ${ }^{4,8}$ Chris L. Fryer, ${ }^{9}$ Nicole M. Lloyd-Ronning ${ }^{\circledR 9,10}$ \\ and Stephan Rosswog ${ }^{11}$ \\ ${ }^{1}$ INAF Osservatorio Astronomico di Roma, I-00040 Rome, Italy \\ ${ }^{2}$ Dip. di Fisica, Universitá di Roma Sapienza, P.le A. Moro, 2, I-00185 Rome, Italy \\ ${ }^{3}$ Dip. di Fisica, Università di Roma Tor Vergata, Via della Ricerca Scientifica 1, I-00133 Roma, Italy \\ ${ }^{4}$ DARK, Niels Bohr Institute, University of Copenhagen, Blegdamsvej 17, DK-2100 Copenhagen, Denmark \\ ${ }^{5}$ Gran Sasso Science Institute, Viale F. Crispi 7, I-67100 L'Aquila, Italy \\ ${ }^{6}$ Division of Physics, Math, and Astronomy, California Institute of Technology, Pasadena, CA 91125, USA \\ ${ }^{7}$ Nikhef, Science Park 105, NL-1098 XG Amsterdam, the Netherlands \\ ${ }^{8}$ Department of Astronomy and Astrophysics, University of California, Santa Cruz, CA 95064, USA \\ ${ }^{9}$ Center for Theoretical Astrophysics, Los Alamos National Laboratory, Los Alamos, NM 87545, USA \\ ${ }^{10}$ Department of Math, Science and Engineering, University of New Mexico, Los Alamos, NM 87544 US \\ ${ }^{11}$ The Oskar Klein Centre, Department of Astronomy, AlbaNova, Stockholm University, SE-106 91 Stockholm, Sweden
}

\begin{abstract}
The combined detection of a gravitational-wave signal, kilonova, and short gamma-ray burst (sGRB) from GW170817 marked a scientific breakthrough in the field of multimessenger astronomy. But even before GW170817, there have been a number of sGRBs with possible associated kilonova detections. In this work, we re-examine these 'historical' sGRB afterglows with a combination of state-of-the-art afterglow and kilonova models. This allows us to include optical/near-infrared synchrotron emission produced by the sGRB as well as ultraviolet/optical/near-infrared emission powered by the radioactive decay of $r$-process elements (i.e. the kilonova). Fitting the light curves, we derive the velocity and the mass distribution as well as the composition of the ejected material. The posteriors on kilonova parameters obtained from the fit were turned into distributions for the peak magnitude of the kilonova emission in different bands and the time at which this peak occurs. From the sGRB with an associated kilonova, we found that the peak magnitude in $H$ bands falls in the range $[-16.2,-13.1]$ (95 per cent of confidence) and occurs within $0.8-3.6 \mathrm{~d}$ after the sGRB prompt emission. In $g$ band instead we obtain a peak magnitude in range $[-16.8,-12.3]$ occurring within the first $18 \mathrm{~h}$ after the sGRB prompt. From the luminosity distributions of GW170817/AT2017gfo, kilonova candidates GRB130603B, GRB050709, and GRB060614 (with the possible inclusion of GRB150101B, GRB050724A, GRB061201, GRB080905A, GRB150424A, and GRB160821B) and the upper limits from all the other sGRBs not associated with any kilonova detection we obtain for the first time a kilonova luminosity distribution in different bands.
\end{abstract}

Key words: gravitational waves - nuclear reactions, nucleosynthesis, abundances - gammaray burst: general.

\section{INTRODUCTION}

Compact binary mergers are the main sources of gravitational waves $(\mathrm{GW})$ in the LIGO-Virgo frequency range and among them binary neutron stars (BNS) and neutron star-black hole (NS-BH) 
systems play a special role since they are also potential sources of electromagnetic radiation. A BNS/NS-BH coalescence in fact could lead to the formation of a BH (or even an NS in BNS case) surrounded by an accretion disc that is expected to power a highly relativistic jet that will produce a short gamma-ray burst (sGRB) lasting few seconds (Eichler et al. 1989; Paczynski 1991; Narayan, Paczynski \& Piran 1992; Mochkovitch et al. 1993; Lee \& RamirezRuiz 2007; Nakar 2007). The sGRB is then followed by a fading synchrotron cooling afterglow from the shock of the jet with the external medium. This afterglow is visible in X-rays, optical and radio for days to months after the initial prompt gamma-ray emission (Sari, Piran \& Narayan 1998).

Moreover, during the merger, a fraction of the NS matter can be ejected from the system either by tidal torques or hydrodynamical forces. This component of unbound matter, usually called 'dynamical ejecta', is highly neutron rich and therefore is a natural site for the synthesis of $r$-process elements (Lattimer \& Schramm 1974, 1976), whose radioactive decay can heat the ejecta and power a thermal ultraviolet/optical/near-infrared transient known as kilonova (or macronova) (Li \& Paczynski 1998; Metzger et al. 2010; Roberts et al. 2011; Kasen et al. 2017). Contrary to the sGRB prompt and afterglow emission, this transient is expected to be broadly isotropic. This means that in principle after every BNS merger which eject a sufficient amount of matter and every NS-BH mergers leading to the NS disruption, ${ }^{1}$ we could expect to observe a kilonova regardless of the orientation of the system (Roberts et al. 2011).

This characteristic, along with a peak in the bolometric light curve of $10^{40}-10^{41} \mathrm{erg} \mathrm{s}^{-1}$ at a few hours/days after the merger, makes kilonovae optimal targets for an observational campaigns of GW's electromagnetic counterparts (Metzger \& Berger 2012). The observational features of kilonovae depend mainly on the mass, velocity, and composition of the ejecta. These parameters are in turn correlated with the equation of state (EOS) of neutron stars (NS) and the mass ratio of the binary (Bauswein, Baumgarte \& Janka 2013; Piran, Nakar \& Rosswog 2013; Abbott et al. 2017a Bauswein et al. 2017; Dietrich \& Ujevic 2017; Radice et al. 2018a). A further crucial parameter is the matter opacity, which strongly influences the spectral range of the emission, the peak luminosity, and the time at which the peak occurs (Grossman et al. 2014). The matter opacity depends on the fraction of lanthanides (produced in $r$ process nucleosynthesis) within the ejecta, since the bound-bound opacity of these elements dominates all the other contributions. Dynamical ejecta may also consist of more than one component of matter characterized by different lanthanide fractions and thus different opacities. The lanthanide free ejecta would generate a bluer and faster evolving transient known as blue kilonova (Metzger \& Fernandez 2014; Perego et al. 2014) while the lanthanide-rich ejecta would be responsible for the classical red kilonova (Kasen, Badnell \& Barnes 2013). These multiple components arise from different ejection mechanisms: the matter ejected by tidal torques, being particularly neutron rich, is expected to be rich in lanthanides, while that expelled by hydrodynamical forces (i.e. the matter squeezed in the contact interface between the two NS or driven by turbulent viscosity; Radice et al. 2018b) would be lanthanides free, since the increase of temperature due to shock-heating reflects in

\footnotetext{
${ }^{1}$ During an NS-BH merger the NS disruption is not guaranteed. Whether it happens or not depends on the dense matter EOS and on the system's parameters, such as the masses of the compact objects and the BH's spin. In general low NS compactness, low BH masses, and high spins favour the NS disruption (Pannarale \& Ohme 2014).
}

changing the $\beta$-equilibrium in favour of a less neutron-rich mixture (Wanajo et al. 2014; Rosswog 2015).

A further contribution to the kilonova may come from matter expelled from the accretion disc through winds driven by neutrino energy, magnetic fields, viscous evolution, and/or nuclear recombination energy (Fryer, Woosley \& Hartmann 1999; Matteo, Perna \& Narayan 2002; Lee, Ramirez-Ruiz \& Page 2005; Metzger, Piro \& Quataert 2008; Dessart et al. 2009; Lee, Ramirez-Ruiz \& LópezCámara 2009; Fernández \& Metzger 2013; Perego et al. 2014; Siegel, Ciolfi \& Rezzolla 2014; Ciolfi \& Siegel 2015; Just et al. 2015). This component of matter is expelled after the dynamical ejecta and it is expected to travel with lower velocity $(\sim 0.05 c$ against $0.1-0.3 c$ of dynamical ejecta). Its lanthanide fraction decreases with increasing neutrino irradiation from the disc and the merger remnant, which is high if the remnant is a fast spinning $\mathrm{BH}$ and is maximum if the remnant is a long lived NS (Kasen, Fernandez \& Metzger 2015).

The relative contribution of each component depends on the source properties including the binary mass ratio and the nuclear EOS (Rosswog et al. 1999; Bauswein et al. 2013; Hotokezaka et al. 2013; Lehner et al. 2016; Radice et al. 2016; Abbott et al. 2017d; Dietrich \& Ujevic 2017; Siegel \& Metzger 2017).

All of these three electromagnetic components (sGRB, afterglow, and kilonova) described above have been observed (Abbott et al. 2017b; Chornock et al. 2017; Coulter et al. 2017; Drout et al. 2017; Goldstein et al. 2017; Haggard et al. 2017; Hallinan et al. 2017; Kilpatrick et al. 2017; Margutti et al. 2017; Pian et al. 2017; Savchenko et al. 2017; Shappee et al. 2017; Smartt et al. 2017; Tanvir et al. 2017; Troja et al. 2017; Murguia-Berthier et al. 2017b; D'Avanzo et al. 2018; Ghirlanda et al. 2019) following GW170817, the first BNS merger event observed by the LIGO Scientific \& Virgo Collaborations on 2017 August 17 (Abbott et al. 2017a). The kilonova associated with GW170817 (named AT2017gfo) showed a peak in the bolometric luminosity of $\sim$ few $10^{41} \mathrm{erg} \mathrm{s}^{-1}$ in the first $36 \mathrm{~h}$ after the merger and a very rapid spectral evolution from blue to red (Pian et al. 2017; Smartt et al. 2017; Tanvir et al. 2017). Although this event is the first unambiguous detection of a kilonova, a few candidates, appearing as near-infrared excesses emerging late time from sGRB afterglow light curves, have been identified in the recent past. The first to be discovered and probably the most interesting of them was found in association with GRB130602B (Berger, Fong \& Chornock 2013; Tanvir et al. 2013). Subsequently other two candidates have been identified in association with GRB050709 (Yang et al. 2015) and GRB060614 (Jin et al. 2015). Contrary to the case of GW170817/AT2017gfo these claimed detections consist of a single photometric point and the lack of any spectrum makes it impossible to clearly assess the nature of these excesses. In addition, the concurrent $\mathrm{X}$-ray excess in some of these events, e.g. GRB080503, GRB130603B, suggest that the near-infrared excess could be explained by shock heating and not kilonova emission (Kasliwal et al. 2017b). Nevertheless, their chromatic nature along with the time and luminosity at which they have been observed makes the kilonova interpretation plausible.

In this article, we are interested in measuring the relative contributions of the afterglow and the kilonova. The kilonova is distinguishable with its nearly isotropic emission, bolometric luminosity, and colour evolution (Metzger et al. 2010; Roberts et al. 2011; Barnes \& Kasen 2013; Kasen et al. 2013; Tanaka \& Hotokezaka 2013; Kasen et al. 2015; Barnes et al. 2016; Metzger 2017). For our analysis, we combine state-of-the-art afterglow and kilonova models and fit them to optical/near-infrared (NIR) short GRB data. We use the optical/NIR data to understand the spectral 
parameters of the afterglow and determine whether there is any excess light from a kilonova. We use a parametrized surrogate model presented in Coughlin et al. (2018b) and based on simulations from Kasen et al. (2017) of AT2017gfo for the kilonova and a structured jet model for short GRBs. We use then the obtained distributions to produce for the first time a kilonova luminosity distribution in different filters. We also calculate for each kilonova event the contribution of $r$-process element local density with an analysis similar to that performed by Abbott et al. (2017d) and compare the results with the Solar system measures.

The paper is organized as follows: in Section 2 we describe our data sample and kilonova and SGRB models employed in the data fitting. In Section 3 we present the results of our analysis, which comprise the distribution of mass, velocity, and lanthanides fraction for all the kilonovae events, the peak luminosity distribution for all kilonovae events as well as the upper limits placed by the kilonovae non-detections, the luminosity distribution of kilonovae in different filters, and the contribution to the local $r$-process elements density for each event. Finally in Section 4 we briefly summarize our analysis and draw the conclusion of our work.

\section{DATA AND ANALYSIS TECHNIQUE}

We begin describing our sGRB sample. This comprises the events GRB130603B (Berger et al. 2013; Tanvir et al. 2013), GRB140903A (Troja et al. 2016), GRB060614 (Zhang et al. 2007; Jin et al. 2015; Yang et al. 2015), GRB050709 (Fox et al. 2005; Hjorth et al. 2005; Covino et al. 2006; Jin et al. 2016), GRB061201 (Stratta et al. 2007), GRB050724A (Berger et al. 2005; Malesani et al. 2007), GRB150101B (Fong et al. 2016; Troja et al. 2018a), GRB080905A (Rowlinson et al. 2010; Nicuesa Guelbenzu et al. 2012), GRB070724A (Berger et al. 2009; Kocevski et al. 2010), GRB160821B (Kasliwal et al. 2017c; Jin et al. 2018), and GRB150424A (Tanvir et al. 2015; Jin et al. 2018). In addition to the short GRBs here, we include measurements from GW170817 (GRB170817A) (Abbott et al. 2017c). This sample is a subset of the Gompertz et al. (2018) sample, which includes all SGRBs with measured redshift $z \leq 0.5$ and from which we selected only the events with an optical/NIR afterglow detected (not just upper limits). This cut in redshift is motivated by the fact that for $z$ $>0.5$ the faint kilonova emission would be unlikely detected by present and upcoming telescope facilities. Nevertheless, this limit is much deeper than the LIGO-Virgo horizon at design sensitivity for BNS and NS-BH mergers (Abadie et al. 2010). We excluded, as Gompertz et al. (2018) did, also GRB061006, GRB071227, and GRB170428 due to their too luminous host galaxies. All the photometric data have been corrected for the Milky Way extinction. In Table A1 the salient information of all the GRBs (and GW170817) in the sample have been summarized. For the cases with a kilonova detection (or claimed detection), the ejecta mass and lanthanide fraction inferred from our analysis are also furnished.

As described above, sGRB afterglows are typically modelled as a decelerating and decollimating relativistic jets producing synchrotron emission. From numerical simulations and the analyses of GW170817, slow-moving cocoon (Nagakura et al. 2014; Kasliwal et al. 2017a; Lazzati et al. 2017; Mooley et al. 2017; Murguia-Berthier et al. 2017b) and Gaussian structured jet (Troja et al. 2017) models seem to be preferred, while a universal jet structure seems to be disfavoured (Kasliwal et al. 2017a; Troja et al. 2017). In the Gaussian structured jet case, energy drops as $E(\theta)=E_{0} \exp \left[-\theta^{2} /\left(2 \theta_{c}^{2}\right)\right]$ up to a truncating angle $\theta_{w}$, where $E_{0}$ is the isotropic equivalent energy and $\theta_{c}$ is the opening angle. In the following, we will use the formalism adopted by Troja et al. (2018b), where the Gaussian jet is implemented as a series of concentric top hat jets and the cocoon as a decelerating shell model which includes the ongoing energy injection. We use the implementation in afterglowpy (Ryan et al., in preparation). This formalism considers also the effect of the viewing angle $\theta_{v}$, which is thus a further parameter of the model. Concerning the other parameters, we denote as $n$ the number density of the homogeneous environment containing the jet and the power-law distribution slope in energy of the electrons undergoing synchrotron emission as $p$. A fraction $\epsilon_{E}$ contains the post-shock internal energy, while a fraction $\epsilon_{B}$ contain the shock-generated magnetic field energy.

For the kilonova model, we use an interpolated surrogate model based on Kasen et al. (2017), which is described in Coughlin et al. (2018b). The model is parametrized by three variables: the ejecta mass $M_{\mathrm{ej}}$, the mass fraction of lanthanides $X_{\text {lan }}$, and the ejecta velocity $v_{\text {ej. }}$. This model provides a state-of-the-art parametrized model to test our analysis method. But it makes a series of assumptions that may ultimately affect our results. It assumes spherical symmetry and a uniform composition and uses multiwavelength radiation transport combined with atomic line data to derive the model. For the isotopes calculated, the atomic data is state-of-the-art. But, at this time, many of the lanthanide opacities have not been calculated and, like other studies, this model uses a few well-calculated opacities as surrogates for the entire set of lanthanides. With multiple ejection processes (dynamical ejecta from tidal disruption, winds from an accretion disc and, if the compact object remains an NS, outflows from NS accretion), the ejecta is likely to have a range of compositions and velocity profiles. In addition, uncertainties in the nuclear physics can produce radioactive isotopes that can significantly alter the radioactive heating, altering the light curve (Zhu et al. 2018). In addition, this model assumes that all the kilonova energy is furnished by the radioactive decay of the nuclides synthesized during $r$-process nucleosynthesis and no kind of central engine (e.g. magnetar, pulsar, fallback accretion) is taken into account. The inclusion of this further contribution could lead to a widening of the distributions of kilonova parameters and in particular to lower values of ejecta mass as found by Matsumoto et al. (2018). These differences are, to a large extent, the cause in the different yield estimates from GW170817 (Côté et al. 2018). For the analyses that follow, we will show examples where the afterglow and kilonova models are fit separately to the data, as well as examples where we add the models together to create joint distributions.

We compare these models to observational data following Coughlin et al. (2017), i.e. randomized sets of light curves are computed for each model, and a $\chi^{2}$ value is calculated between each model and the data. For the kilonova model, the priors are taken to be flat between: $-5 \leq \log _{10}\left(M_{\mathrm{ej}} / \mathrm{M}_{\odot}\right) \leq 0$, $0 \leq v_{\text {ej }} \leq 0.3 \mathrm{c}$, and $-9 \leq \log _{10}\left(X_{\text {lan }}\right) \leq-1$. For the afterglow model, the priors are taken to be flat between: $0 \leq \theta_{v} \leq \pi / 4$, $0 \leq \theta_{c} \leq \pi / 4,0 \leq \theta_{w} \leq \pi / 4,49 \leq \log _{10}\left(E_{0} / \mathrm{erg}\right) \leq 55$, $-4 \leq \log _{10}\left(n / \mathrm{g} / \mathrm{cm}^{3}\right) \leq 0,2.1 \leq p \leq 2.5,-4 \leq \log _{10}\left(\epsilon_{E}\right) \leq 0$ and $-4 \leq \log _{10}\left(\epsilon_{B}\right) \leq 0$. The light-curve fitting code is available at: https://github.com/mcoughlin/gwemlightcurves.

\section{RESULTS}

\subsection{Mass, lanthanide fraction, and luminosity distributions}

We begin with an analysis of GRB130603B to illustrate the method.

Fig. 1 shows the observed data superimposed on different fitted models. Here afterglow only denotes the Gaussian afterglow model 


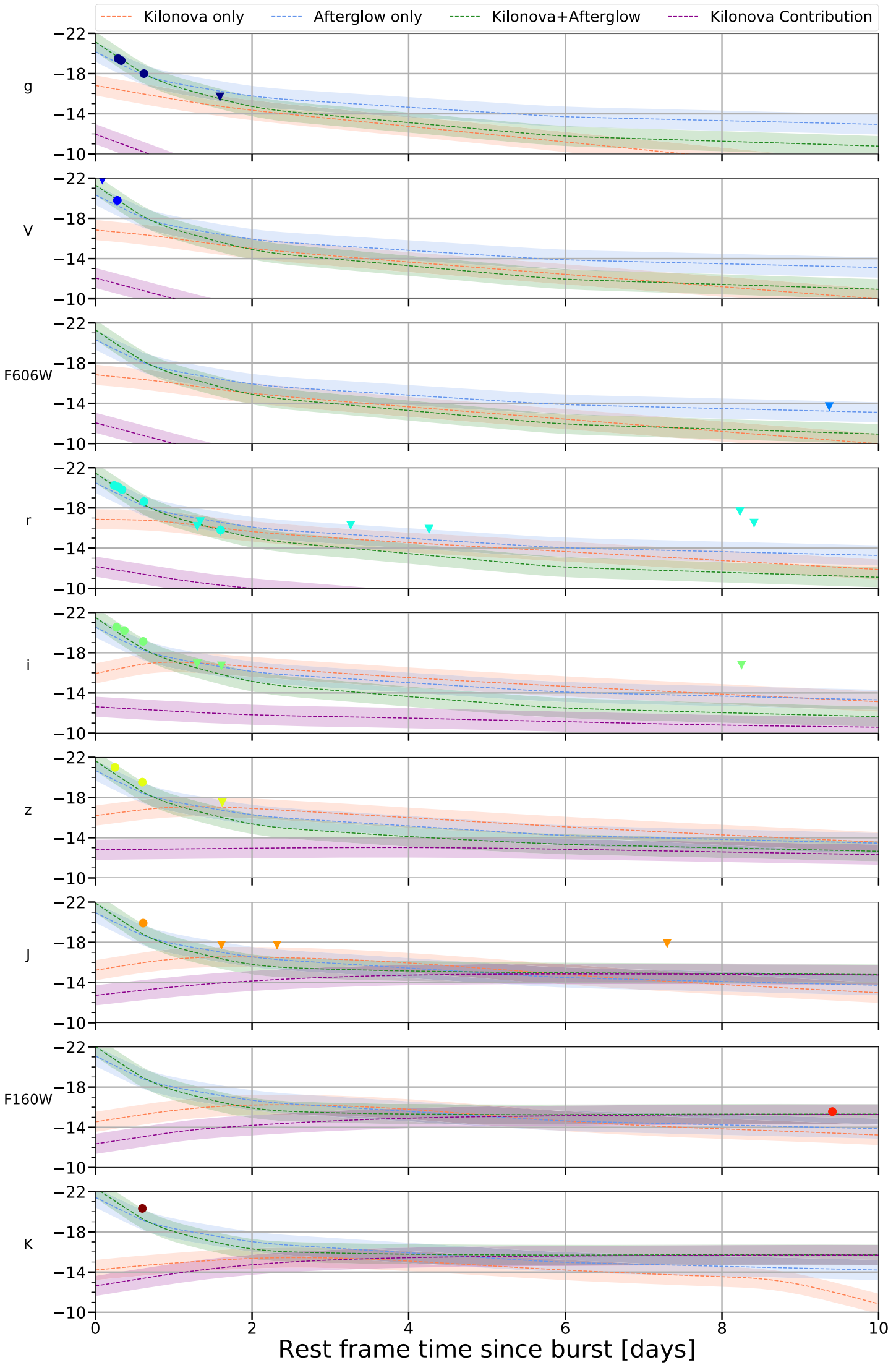

Figure 1. Light curves for the Gaussian afterglow model (Troja et al. 2018b), the kilonova model (Kasen et al. 2017), and a sum of the afterglow and kilonova model for GRB130603B. 'Kilonova part' denotes the kilonova contribution to the 'afterglow+kilonova' model. The shown light curves correspond to a maximum likelihood $\chi^{2}$ fit to the data. All the light curves are expressed in AB absolute magnitudes. The circles denote actual detections while the triangles are upper limits. 

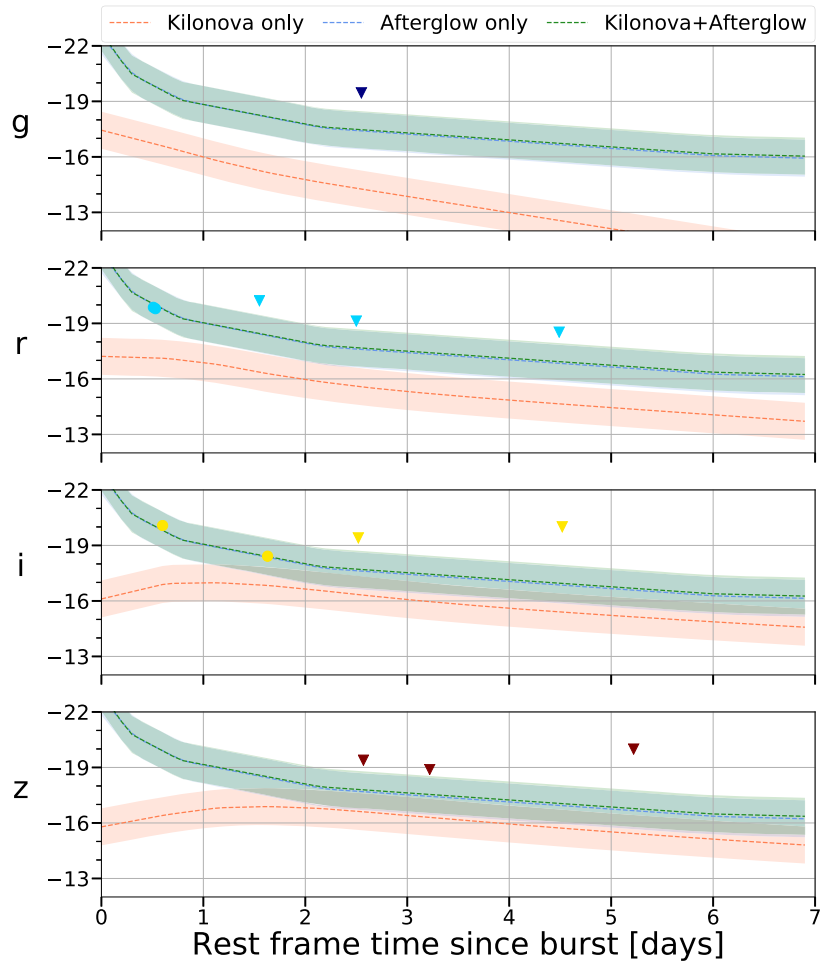

Figure 2. Same as Fig. 1 for GRB140903A. Afterglow and Kilonova+Afterglow models superimposed exactly and are not distinguishable.

of Troja et al. (2018b), kilonova only the kilonova model of Kasen et al. (2017), and kilonova + afterglow the combination between the two models. Adding a kilonova contribution to the afterglow causes an increase in the optical flux at early times and, as a result, predicts less flux from the afterglow at later times (compared to the afterglow only model). The additional (relative to the afterglow) contribution necessary from the kilonova to account for the observations is shown by the purple line (denoted as kilonova contribution).

In this figure we can see that the kilonova in addition to the afterglow is required to fit the data, as noted at the time of detection (Berger et al. 2013; Tanvir et al. 2013). We find that the estimate of the ejecta mass based on the joint analysis is $M_{\mathrm{ej}}=7.46_{-7.29}^{+43.97} \times$ $10^{-2} \mathrm{M}_{\odot}$.

A different case, shown in Fig. 2, is that of GRB140903A, where the afterglow fit dominates the performance of the fit. If a kilonova is present here its light curve is completely buried in the afterglow light curve and any upper limit on ejecta mass would be too high to be informative. In fact the fit of the kilonova model results in $M_{\mathrm{ej}} \leq 7.46 \times 10^{-1} \mathrm{M}_{\odot}$.

The final scenario is represented by GRB150101B and is shown in Fig. 3. In this case the afterglow, the kilonova and the afterglow+kilonova fit perform equally, which means that although we cannot claim a kilonova detection we can put an informative upper limit on the ejecta mass. This measure is equal to $M_{\text {ej }}=3.17_{-1.56}^{+3.12} \times 10^{-2} \mathrm{M}_{\odot}$.

We want to use our analysis of the individual short GRBs to make constraints on the luminosity and ejecta mass of the kilonovae. For some of the short GRBs, such as GRB140903A and GRB050724A, the photometry is such that no (informative) limits on kilonova emission are possible; in other words the analysis gives back the parameters priors. The ones of most interest to us are the ones which
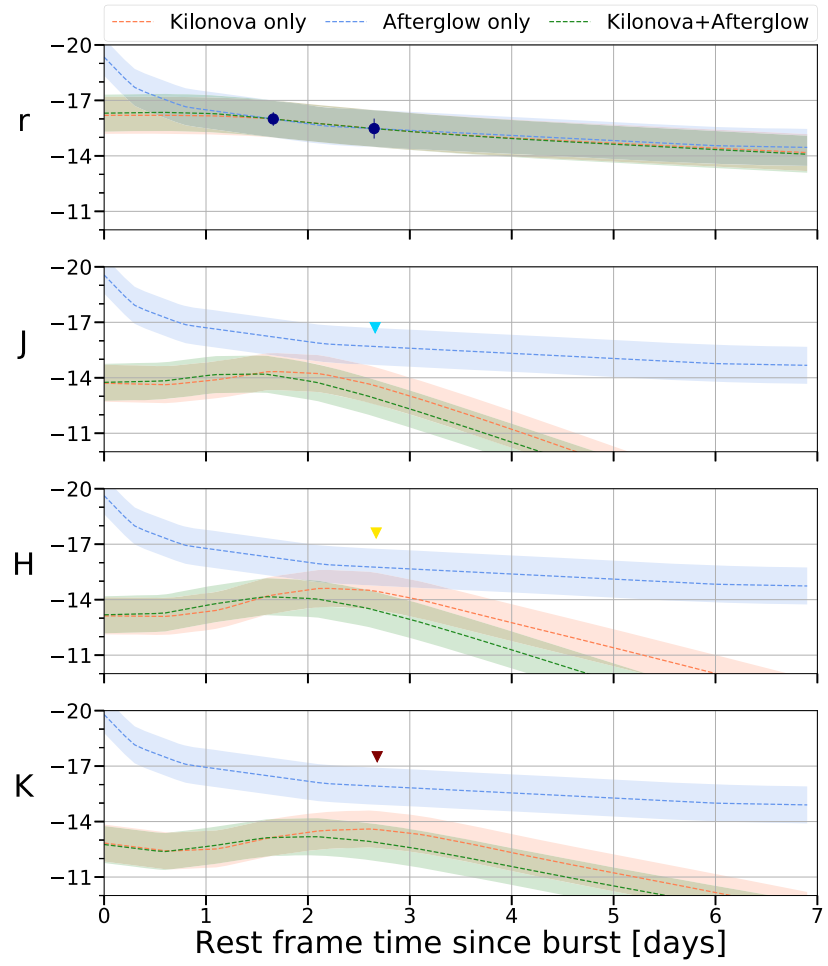

Figure 3. Same as Fig. 1 for GRB150101B.

provide some limits like GRB061201 and GRB080905A (as also noted by Gompertz et al. 2018), or even (claimed) detections of kilonovae. These include GRB150101B, GRB050709, GRB130603B, GRB060614, and GW170817/GRB170817A.

We first show the light curves predicted by the fitting analysis in Fig. 4. These light curves are similar in concept to those in Gaussian Process Regression, where the light curves span the possible extrapolations based on the model, which is in this case the kilonova surrogate model. Only in the case of GRB130603B is the afterglow model added because we need both the afterglow and kilonova components to fit the data. In the plot, the dashed lines show the median light curve, while the shaded intervals show the 95 per cent intervals.

Fig. 5 shows the posteriors of the $M_{\mathrm{ej}}, v_{\mathrm{ej}}$, and $X_{\mathrm{lan}}$ for the events that we regard as a real kilonova detection, where we also included the recently claimed blue kilonova associated to GRB150101B (Troja et al. 2018a). They are both broadly consistent in this measurement to what was found for GW170817. This is not an accident, as there is a significant selection effect in this analysis. Some afterglows return the priors (see Fig. A1 for the posterior distributions of the afterglow's parameters), given the significant energies involved; their light curves are not informative. This is the subset with low enough afterglow energies to be consistent with the energies we expect from kilonovae. Perhaps most interesting that no observations are consistent with measurements lower than $\approx 0.05 \mathrm{M}_{\odot}$. These large masses are commonly thought to be less likely to be produced by dynamical ejecta (e.g. Bauswein et al. 2013; Hotokezaka et al. 2013; Rosswog 2013; Dietrich et al. 2015). Instead magnetized or neutrino-irradiated wind from a long-lived hypermassive NS remnant prior to its collapse to a black hole is usually invoked (Metzger, Thompson \& Quataert 2018). In general, GRB130603B has the broadest range of possible parameters for a few reasons. As stated previously, GRB130603B is the only one 

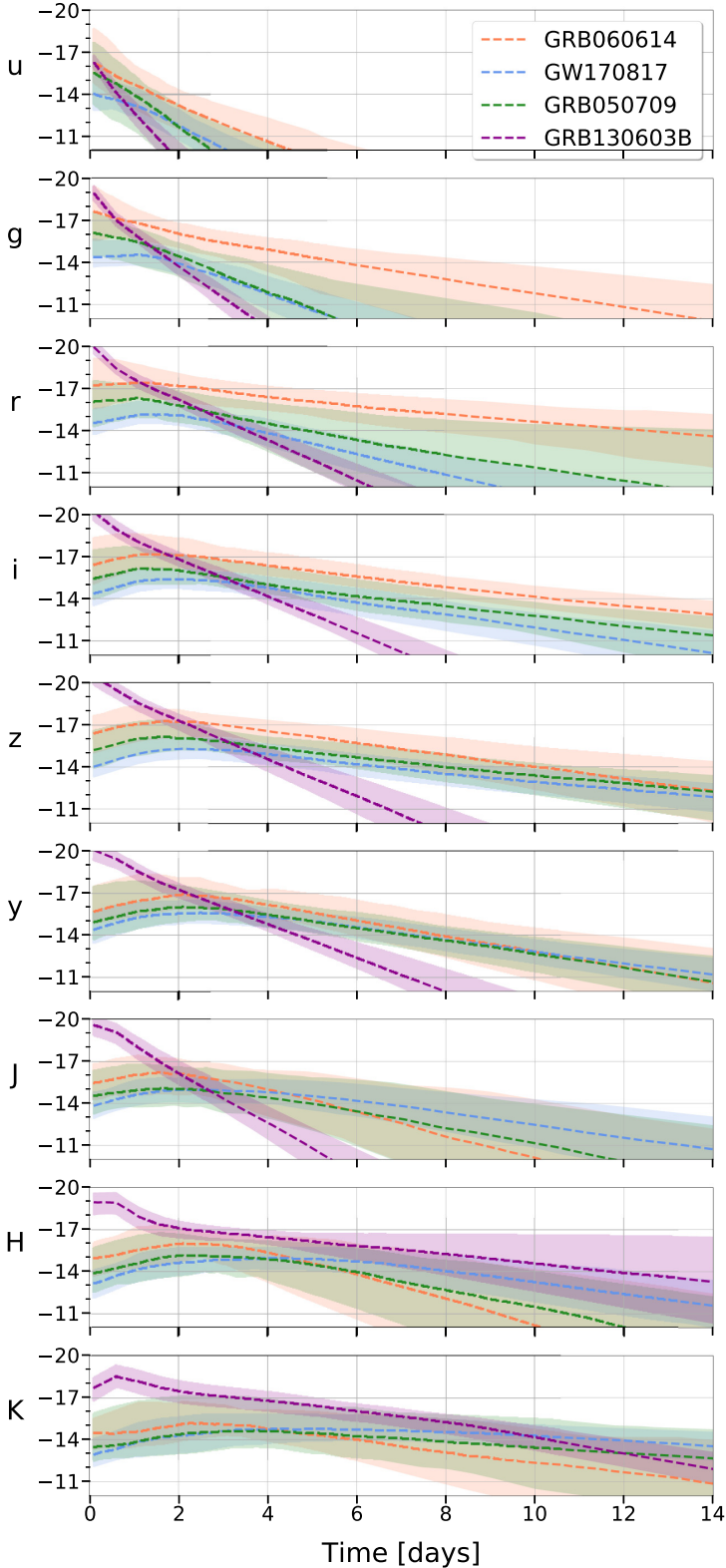

Figure 4. Light curves obtained by the fitting procedure for the kilonova events. The light curves are those of the surrogate kilonova model with the exception of GRB130603B where a kilonova+afterglow model has been employed. The dashed lines show the median light curve, while the shaded intervals show the 95 per cent intervals. The numbers to the left of the $y$-axis show the passbands of the observations.

where we include the afterglow model as well. In addition, the main contribution of the kilonova model is to improve the fit to the final data point at about $9.5 \mathrm{~d}$. For this reason, the posteriors are driven by any kilonova parameters that pass through this set of data points. These are required to achieve a light curve sufficiently red to reach a magnitude brighter than $m_{\mathrm{AB}}=-16$ and blue such that it is dimmer than $m_{\mathrm{AB}}=-14$. This event is more consistent with large amounts of red ejecta, which could originate from an accretion disc outflow (e.g. Metzger \& Fernandez 2014; Perego et al. 2014), just as the blue ejecta.

The distributions of $M_{\mathrm{ej}}, v_{\mathrm{ej}}$, and $X_{\mathrm{lan}}$ have been turned into distributions for time of the peak and peak magnitudes in different
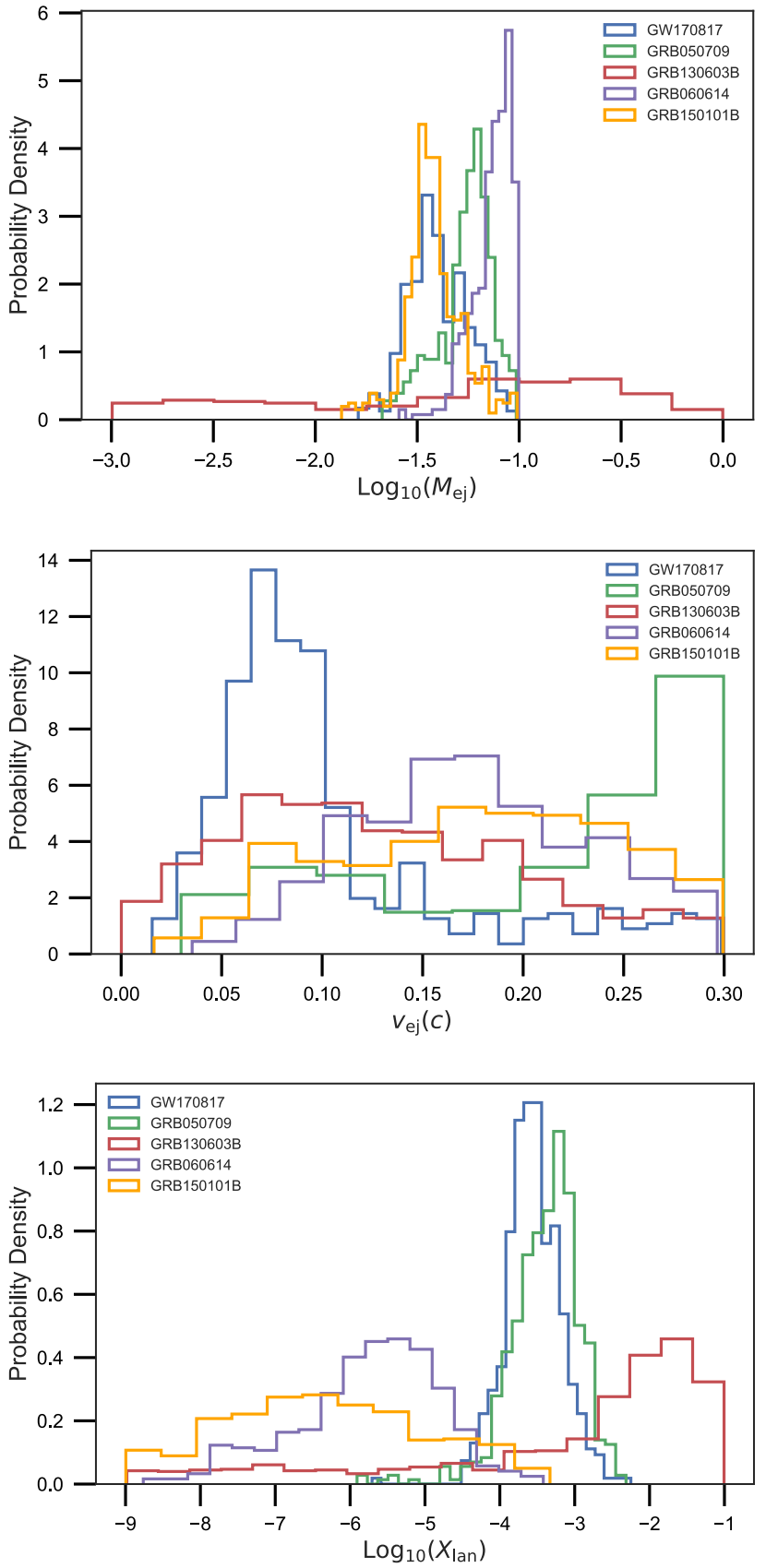

Figure 5. Ejecta mass (top), ejecta velocity (middle), and lanthanide fraction (bottom) estimates based on the GRB sample with an associated kilonova considered in this paper.

filters. To this aim, first a distribution of opacities $k$ has been obtained from $X_{\text {lan }}$ using a log-linear relation between the two parameters described by the equation:

$$
\begin{aligned}
k\left(X_{\mathrm{lan}}\right) & =\left\{\begin{array}{ll}
m \log _{10}\left(X_{\mathrm{lan}}\right)+q & X_{\mathrm{lan}} \geq 10^{-6} \\
k_{0} & X_{\mathrm{lan}} \leq 10^{-6}
\end{array},\right. \\
m & =\frac{k_{1}-k_{0}}{\log _{10}\left(X_{\operatorname{lan}, 1}\right)-\log _{10}\left(X_{\mathrm{lan}, 0}\right)}, \\
q & =\frac{k_{0} \log _{10}\left(X_{\mathrm{lan}, 1}\right)-k_{1} \log _{10}\left(X_{\mathrm{lan}, 0}\right)}{\log _{10}\left(X_{\mathrm{lan}, 1}\right)-\log _{10}\left(X_{\mathrm{lan}, 0}\right)},
\end{aligned}
$$


where $k_{0}=0.1 \mathrm{~cm}^{2} \mathrm{~g}^{-1}, k_{1}=10 \mathrm{~cm}^{2} \mathrm{~g}^{-1}, X_{\text {lan, } 1}=10^{-1}$, and $X_{\text {lan, }, 0}=10^{-6}$. This prescription ensures the opacity to be equal to $k_{0}$ when $X_{\text {lan }}=X_{\text {lan }, 0}$ and rise logarithmically to $k_{1}$ at $X_{\text {lan }}=X_{\text {lan, } 0}$.

Then opacities, masses, and velocities of the ejecta have been turned into kilonova multicoloured light curves following the method/model outlined in Metzger (2017). Although this is a simple analytical model, it reproduces results accurate enough for our analysis. For each light curve in different filters the peak of the luminosity, along with the time at which it occurs, has been obtained. We report our results in Figs 6 and A2.

Fig. 6 shows for the most interesting events (those of Fig. 5) the distribution of peak time (top panel) and peak absolute $A B$ magnitude (bottom panel) for the filters $g$ (blue) and $H$ (red). The white dot represents the median of the distribution for the given event. The black bars and lines mark, respectively, the interquartile range and the 95 per cent of confidence interval of the distribution. In Fig. 7 we report the same results for the whole sample.

In the Appendix, in Fig. A2, we present in a 2D peak magnitudepeak time space the probability density distribution (within a 68 percent of confidence interval) in the central panel (again for the filter $g$ and $H$ in blue and red, respectively) to highlight the correlation between the two parameters. These distributions have been drawn smoothing the discrete data with a Gaussian kernel based density estimation. The top and right-hand panels show the marginalized distributions of peak time and peak magnitude, respectively.

Figs 6 and A2 show that in all cases the peak time of the emission in $g$ filter lies within few hours after the merger and within the first $3 \mathrm{~d}$ from the merger/GRB prompt emission in the $H$ filter. The $H$ peak magnitude is expected to lie in the range $[-16.2$, -13.1] (95 per cent of confidence), while the $g$ filter distribution is broader with a peak magnitude laying in the range $[-12.3,-16.8]$. Events GRB060614 and GRB150101B show a double peaked $g$ luminosity distribution with the smaller peak below the median $H$ luminosity. GRB150101B shows also a dominant blue component (referring to the median of the distribution) that results from the low inferred lanthanide fraction. It is worth noticing that recently Troja et al. (2018a) found evidence for a blue kilonova arising from the early time $(t \sim 2 \mathrm{~d})$ ultraviolet/optical light curve of the GRB150101B afterglow. Although we do not find any firm evidence of a kilonova for this GRB, its contribution is not ruled out and still consistent with the result of our analysis. Moreover, the authors found for the kilonova associated with GRB150101B, an ejecta mass $M_{\mathrm{ej}}>0.02 \mathrm{M}_{\odot}$ and an opacity $k \sim 1 \mathrm{~cm}^{2} \mathrm{~g}^{-1}$ (equivalent to $X_{\text {lan }} \sim 10^{-6}$ according to equation 1$)$ both consistent with our results (see Table A1).

Among all the events GRB130603B (the prototype of an afterglow+kilonova fit) shows the largest uncertainties both in peak time and peak magnitude distributions. This follows from the very large inferred distributions of mass and lanthanide fraction (see Fig. 5), which span the full parameter space.

If we look at the Fig. A2 we can see that GW170817 and GRB050709 manifest (in the $H$ filter at least) a correlation between peak time and peak magnitude, with the higher luminosities having the lower peaking time. This correlation is absent in the other cases. Again we can interpret this trend in view of Fig. 5, where it is worth noticing that the two cases considered above are those with the lowest dispersion both in mass and lanthanide fraction. When these parameters are well constrained it is thus natural to expect from the model the event with higher luminosities to peak earlier in time. We expect to observe this kind of trend in future observations with a less limited data set.

\subsection{The kilonova luminosity distribution}

We used the distribution of the peak luminosities in Fig. 7 to build a set of luminosity distributions for each spectral band. We consider first the real kilonovae events, namely GW170817, GRB130603B, GRB050709, and GRB060614 with the eventual inclusion of GRB150101B. Moreover, recently Rossi et al. (2019) claimed a kilonova association also for GRB050724A, GRB061201, GRB080905A, GRB150424A, and GRB160821B, so also they are eventually included. ${ }^{2}$ For each of these events from the peak magnitude distribution in the chosen filter we took the median, the 5th, and the 95th percentiles of the distribution. In this way for each event the distribution is reduced to three values representing the median, the upper, and lower limits on kilonova peak magnitude. Now we turn to all the other events that are not associated to any kilonova. For these cases we take only the 5th percentile of the distribution as the peak magnitude upper limit and we set the median and the lower limit to an infinity magnitude (corresponding to 0 luminosity). In this way for the whole sample we obtain three sets of values that we use to draw three different cumulative distribution functions representing the kilonova luminosity distribution in three limiting cases: the optimistic case, obtained from the upper limits, the pessimistic case obtained from the lower limits, and the median case obtained by the median of the distributions. Whether our luminosity distribution represents also a proper luminosity function of kilonovae depends on the selection effects of our sample. The selection effect in our case is represented by the detection of an afterglow associated to a kilonova. In this way, our luminosity distribution would correspond to the luminosity function for the kilonovae associated with an observed afterglow (which means on-axis orientation and systems with non-choked jets). It is worth noticing that the luminosity function defined in this way is the cumulative distribution of peak magnitude for each event. Therefore, according to this definition, it is not deconvolved from the event rate density nor divided by the comoving volume.

In Fig. 8 we show the luminosity distributions in $g$ and $H$ bands, while the results in all the other bands are reported in Appendix in Fig. A3. Due to the recent claim of a kilonova associated to the event GRB150101B and considering also the fact that even in this analysis the light curve of this event can be fitted by a kilonova model, we decided to repeat the same analysis promoting GRB150101B as a real detection. We promoted also the five events found by Rossi et al. (2019). Since all these claims have been made only recently and the kilonovae differs from the previous kilonovae associated to $\mathrm{sGRBs},{ }^{3}$ we found it useful to include these events separately, in order to allow the comparison of luminosity distributions with and without the inclusion of these events as real kilonova detections. The luminosity distributions obtained with the inclusion of GRB150101B are shown in Figs 9 and $\mathrm{A} 4$.

The luminosity distribution that we obtained allowed us to estimate the exposure time needed for a given telescope facility to detect a kilonova in a given band at a given time. Consider for example the upper limit distribution (dashed line) in Fig. 8. We can see that in $g$ and $H$ bands, the 50 per cent of the events

\footnotetext{
${ }^{2}$ All these five events have been fitted with an afterglow+kilonova model. ${ }^{3} \mathrm{GRB} 150101 \mathrm{~B}$ is bluer in colour, while the claim of a kilonova presence for GRB050724A, GRB061201, GRB080905A, GRB150424A, and GRB160821B is due to the observation of an anomalous shallow decay instead of an excess in the afterglow light curve.
} 


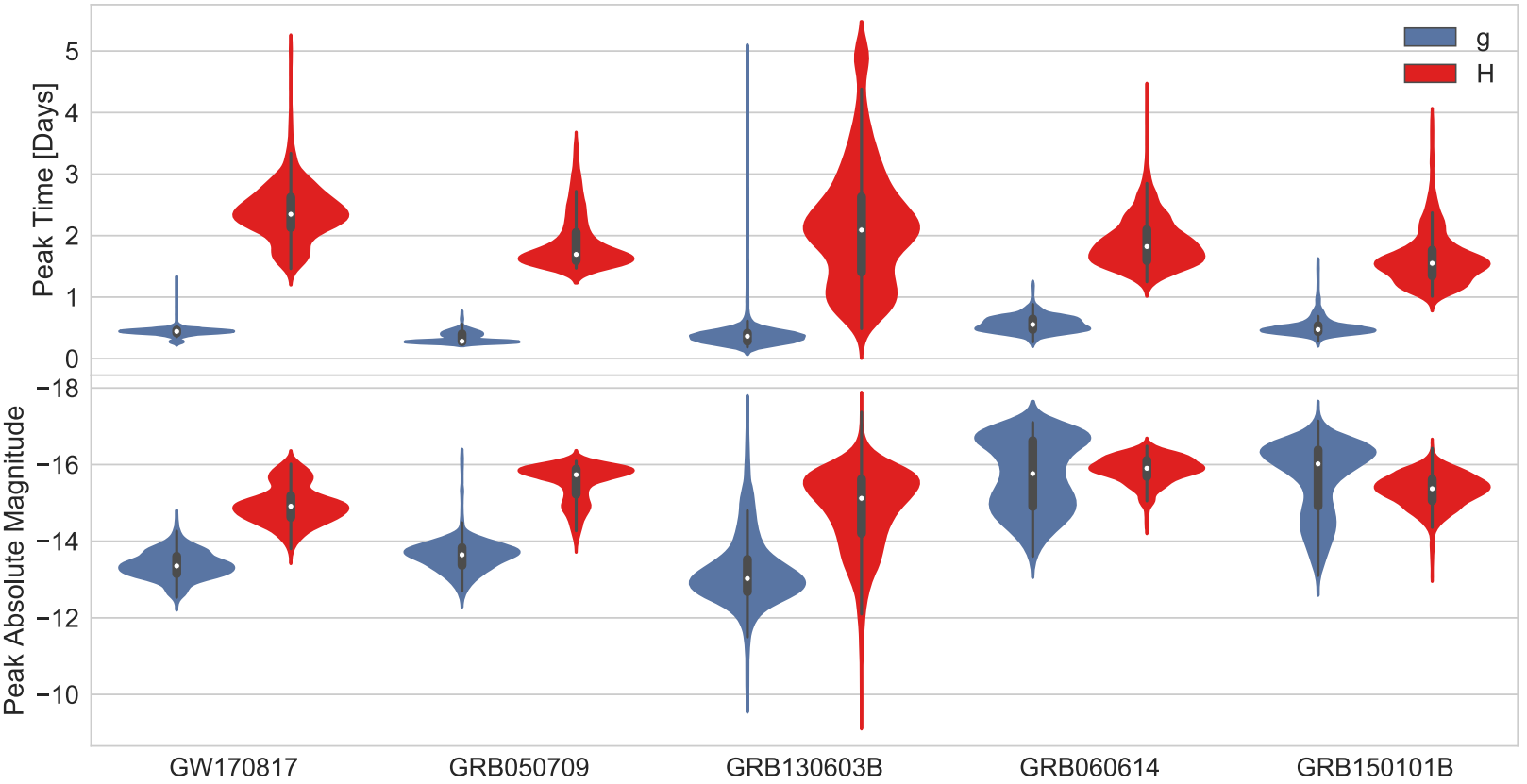

Figure 6. Violin plot showing the peak time (top) and peak magnitude (bottom) for all the events considered in the filters $\mathrm{g}$ (blue) and $\mathrm{H}$ (red). The white dots, black bars, and black lines represent, respectively, the median, the interquartile range, and the 95 per cent confidence interval of the distributions. In this plot only the events that lead to the most constraining measures/upper limits on luminosity distribution are shown. For a plot that include the whole sample see Fig. 7.

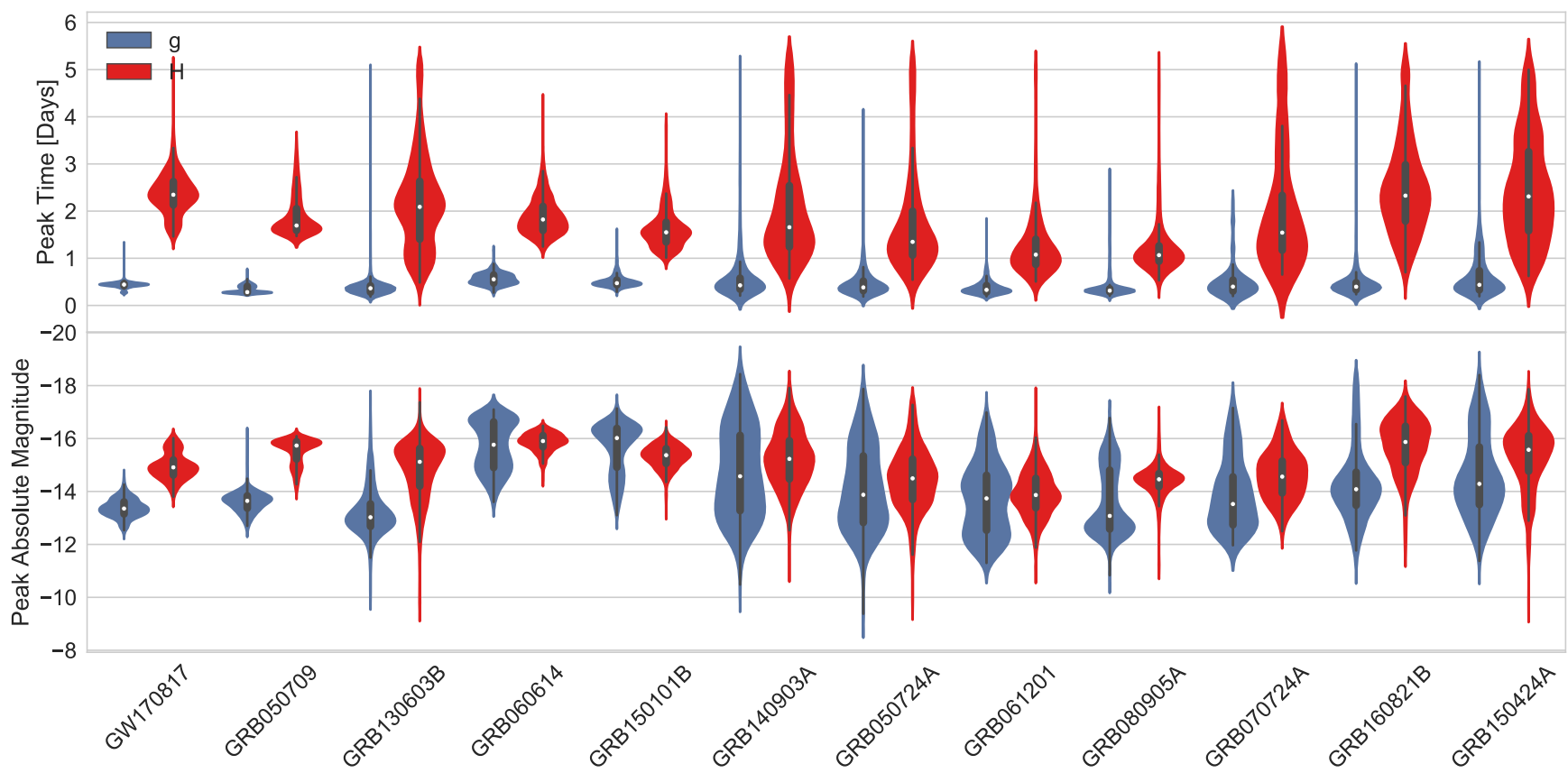

Figure 7. Same as Fig. 6 but with all the events in our sample.

are expected to be fainter, respectively, than -16.5 and $-16 \mathrm{AB}$ absolute magnitude. We can take these values as a benchmark to compute the exposure time needed to detect the 50 percent of the events according to optimistic luminosity distribution. If we consider kilonovae at a fiducial distance of $200 \mathrm{Mpc}$, these values translate to an apparent $\mathrm{AB}$ magnitude of 20.0 and 20.5, respectively. Moreover, in $g$ band the transient is expected to peak within the first $18 \mathrm{~h}$ after the merger/sGRB prompt, while in $H$ band the peak is going to occur between the first and the fourth day.
Using the public Exposure Time Calculators (ETC) of GEMINI ${ }^{4}$ we estimated an exposure time of $\sim 11.5 \mathrm{~s}$ with the instrument GMOS to detect this magnitude in $g$ filter with a signal-to-noise ratio $(\mathrm{S} / \mathrm{N})=20$ within the first day of observation. In the subsequent $4 \mathrm{~d}$, the source could be observed with the instrument NIRI in $H$ filter with 30 exposures each of $\sim 8 \mathrm{~s}$ (equivalent to a total integration

${ }^{4} \mathrm{http} / / / w w w . g e m i n i . e d u / s c i o p s / i n s t r u m e n t s /$ integration-time-calculators 

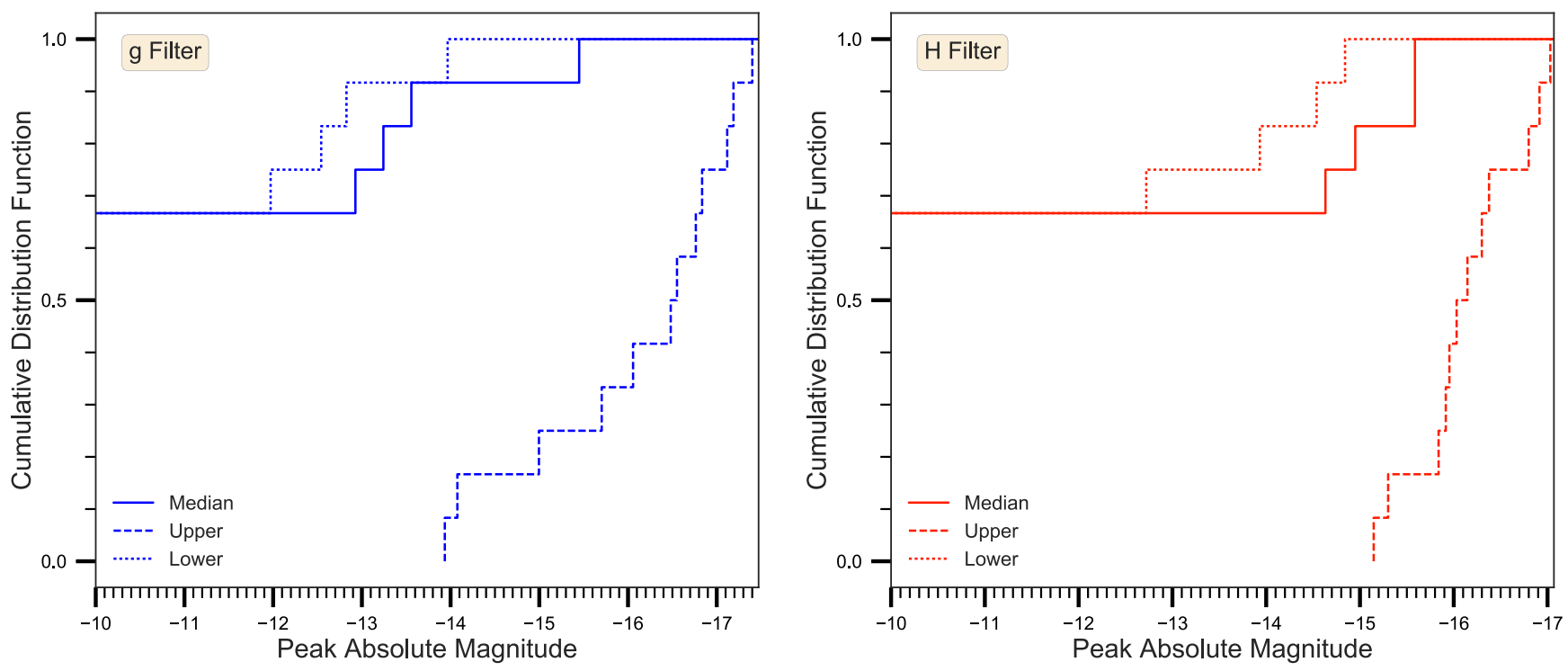

Figure 8. Kilonova luminosity distribution in $g$ (left-hand panel) and $H$ (right-hand panel) filters. The solid, dashed, and dotted lines are obtained from the median, the upper, and the lower limits of the distribution, respectively.
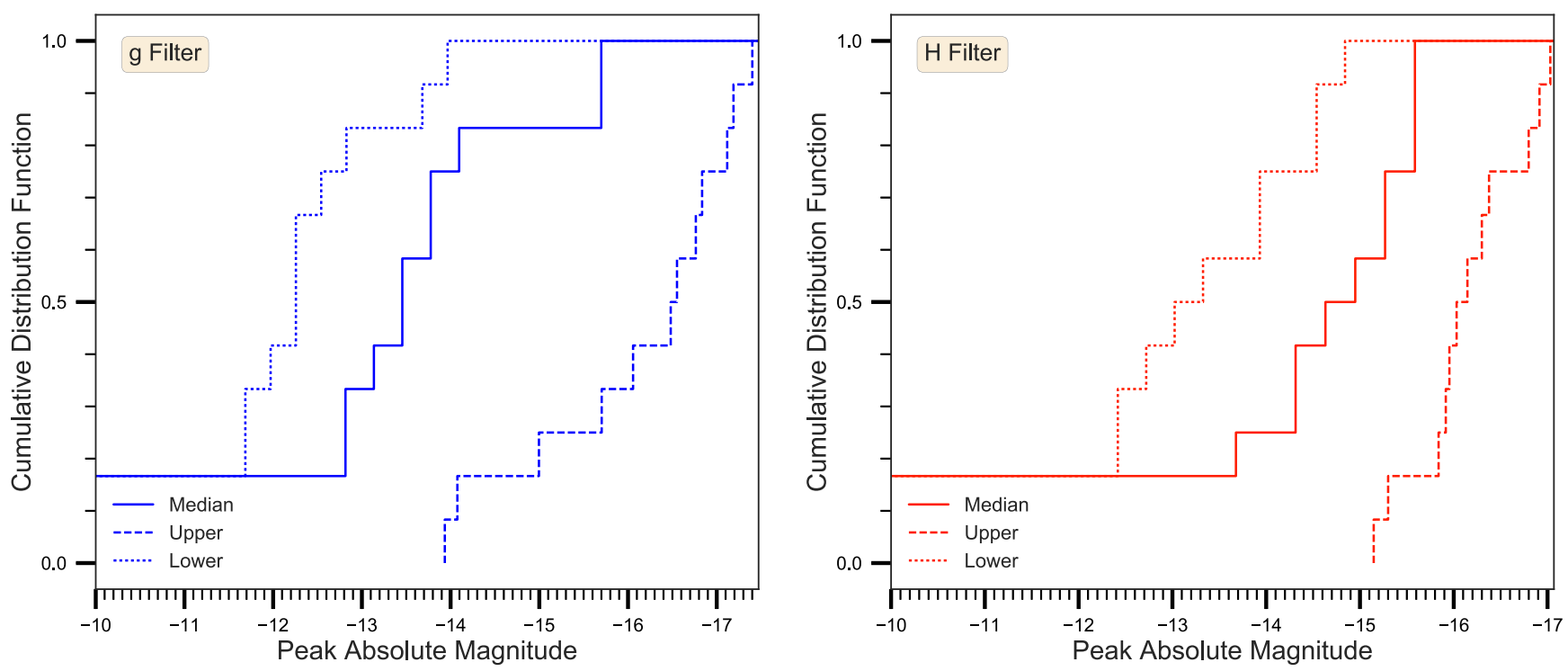

Figure 9. Same as Fig. 8 but here we promoted GRB150101B, GRB050724A, GRB061201, GRB080905A, GRB150424A, and GRB160821B to kilonova events.

time of $240 \mathrm{~s}$ ) to reach a $\mathrm{S} / \mathrm{N}=20$. We repeat the same exercise for VLT. ${ }^{5}$ In this case, the kilonova can be observed in $g$ band within the first $16 \mathrm{~h}$ using FORS2 imager with a single exposure lasting $\sim 1.2 \mathrm{~s}$ (value obtained with input magnitude of 20.0 in $B$ filter as a proxy). In $H$ band instead the transient can be observed with HAWK-I imager with two exposures of $\sim 35 \mathrm{~s}$ for a total exposure time of $70 \mathrm{~s}$. For both GEMINI and VLT a typical airmass of 1.5 has been considered in this calculation.

\subsection{Local density of $r$-process elements}

Finally we repeat the analysis of Abbott et al. (2017d) to estimate the average dynamically ejected local $r$-process material density $\rho_{\text {rp }}$ for

${ }^{5}$ https://www.eso.org/observing/etc/ the events GW170817, GRB050709, GRB151010B, GRB130603B, GRB060614 and GRB050724A, GRB061201, GRB080905A, GRB150424A, GRB160821B. The average local density is calculated according to the formula:

$\rho_{\mathrm{rp}} / f_{\mathrm{rp}}=M_{\mathrm{ej}} \mathcal{R} \frac{\int_{0}^{t_{H}} \int_{0}^{t} \dot{\rho}_{*}(\tau) p_{\text {delay }}(t-\tau) \mathrm{d} \tau \mathrm{d} t}{\int_{0}^{t_{H}} \dot{\rho}_{*}(\tau) p_{\text {delay }}\left(t_{H}-\tau\right) \mathrm{d} \tau}$,

where $f_{\text {rp }}$ is the fraction of dynamical ejecta matter converted in $r$-process elements, $\mathcal{R}$ is the present day merger rate, $t_{H}$ is the Hubble time, $\dot{\rho}_{*}(t)$ is the star formation rate of Madau \& Dickinson (2014), $p_{\text {delay }} \propto 1 / t$ is the distribution of delay time between the BNS formation and its merger (O'Shaughnessy, Belczynski \& Kalogera 2008; Dominik et al. 2012). As in Abbott et al. (2017d) integrating over the cosmic history a $\Lambda$ CDM cosmology with parameters in Planck Collaboration XIII (2016) have been assumed. 
Here we sample from $M_{\mathrm{ej}}$ distributions for the single event, while for the present day rate we sample over a lognormal distribution with a 90 per cent confidence in the range $[360,4730] \mathrm{Gpc}^{-3} \mathrm{yr}^{-1}$ (consistent with the rates inferred from GW170817 (Abbott et al. 2017a). Our results are reported in Fig. 10, where we report on the left-hand side also the mass fraction of $r$-process elements calculated as $Z_{\mathrm{rp}} / f_{\text {rp }} \equiv\left(\rho_{\mathrm{rp}} / f_{\mathrm{rp}}\right) / \rho_{*}$, with $\rho_{*}=\int_{0}^{t_{H}} \dot{\rho}_{*} \mathrm{~d} t$. It is worth noting that the fractions $X_{\text {lan }}$ and $Z_{\mathrm{rp}}$ denote two different quantities: $X_{\text {lan }}$ is the lanthanide fraction in the merger ejecta, while $Z_{\mathrm{rp}}$ is the average mass fraction of $r$-processes in all elements (lanthanides included) in the present day Universe, calculated assuming that all the BNS mergers contribute to the enrichment with the $M_{\mathrm{ej}}$ of the given event.

The red band here denotes the $r$-process elements mass fraction from Solar system observations (Arnould et al. 2007).

It is worth noticing that the average local density obtained from GW170817 is about an order of magnitude higher than that obtained by Abbott et al. (2017d). This discrepancy is due to the different ejecta mass distribution employed in this work (obtained from light-curve fitting), that is about one order of magnitude higher than that used in Abbott et al. [2017d; obtained from the BNS masses distributions plus (Dietrich \& Ujevic 2017) fitting formula]. Nevertheless our results are still (marginally) consistent with the Solar system measured mass-fractions and illustrate the uncertainties associated with deriving accurate ejecta masses given primarily our lack of understanding of $r$-process opacities. In all cases, our masses are above the stringent minimum mass requirements derived from low-metallicity stars in the Universe (Shen et al. 2015; Macias \& Ramirez-Ruiz 2018).

\section{SUMMARY}

Our analysis is the first study using the latest models of AT2017gfo and presents posteriors of $M_{\mathrm{ej}}, v_{\mathrm{ej}}$, and $X_{\mathrm{ej}}$ for the 'historical' sGRBs. In general, both the absolute magnitude predictions and the colour evolution of the kilonovae allow for the differentiation of their contribution from the afterglow.

While GRB130603B was the first short GRB with evidence for a kilonovae followed by GRB050709 and GRB060614, other short GRBs (GRB061201, GRB080905A, and marginally GRB160821B) provide constraints on $r$-process rich ejecta contributions to those light curves. GW170817, the first joint GW-EM detection, provides tighter constraints than GRB130603B both in peak time and luminosity and we expect to observe more events like this in the near future. GRB130603B and GW170817 are in our sample the real kilonova detections, for which our analysis predicts a dominant $H$ over $g$ filter luminosity. In the other events that provide upper limits the $H$ luminosity is dominant as well, with the only exception of GRB150101B.

Considering both real detections and upper limits, our analysis identify so far an $H$ filter peak magnitude in the range of $[-16.2$, -13.1] (along with a $H$ band peak time in range $[0.8,3.6]$ days). We use our sample of nearby $(z<0.5)$ sGRBs that comprise both events with and without a kilonova candidate to draw the first kilonova luminosity distribution in literature in different frequency bands. We build three different limiting luminosity distributions corresponding to the median, lower, and upper limiting values of the peak luminosity distributions. Our results obtained considering the kilonova candidates GRB130603B, GRB050709, and GRB060614 as real kilonovae (Fig. 8) show that in the $H$ filter half of the events are below the -16 th mag in the optimistic case (dashed line) while in the median (solid line) and pessimistic case (dotted line) about the 64 percent of the events are below the -14.6 and $-12.7 \mathrm{mag}$, respectively. Including GRB150101B, GRB050724A, GRB061201, GRB080905A, GRB150424A, and GRB160821B as real kilonovae reduces the difference between the lower and median distribution with the upper distribution, which is in fact a consequence of the fact that in our sample, we have more upper limits than real kilonovae events. In this case the median and the lower distributions result in about 17 percent of the events below $-13.8 \mathrm{mag}$ and $-12.4 \mathrm{mag}$, respectively, while the upper limit is unchanged by construction. For the luminosity distribution in $g$ filter, we observe that the upper case predict half of the events fainter than $-16.3 \mathrm{mag}$, while the lower and the median case predict the 67 per cent of events below the -12.9 and -11.7 mag, respectively. Including GRB150101B, GRB050724A, GRB061201, GRB080905A, GRB150424A, and GRB160821B we obtain that 17 per cent are fainter than -12.8 and -11.7 mag in the median and lower distributions, respectively. We expect that future observations of kilonovae will help to reduce the uncertanities between the three limiting distributions considered here.

The results obtained in this work can be used to predict the absolute magnitude and colour of future events, and inform the search strategies that will be used to detect them. This could include using the predictions and the 3D skymaps to allocate exposure times sufficient to make detections (see e.g. Salafia et al. 2017 and Coughlin et al. 2019). In this way, the kilonova detections can be used as benchmarks for future searches. Further statistical samples will enable making constraints on the progenitor system properties, including the mass ratio and EOS, based on the light curves alone (Coughlin et al. 2018a). Moreover, under the assumption that all BNS are the progenitors of sGRBs, the mergers can be used to constrain their overall contribution to the $r$-process in the Universe (Abbott et al. 2017d).

Future observations, coupled with more detailed theory models, will allow us to place more stringent constraints on the kilonova peak luminosity distribution. Moreover they will allow us to answer the following questions:

(i) Are the kilonovae produced by NS-NS mergers different from those produced by NS-BH mergers (if any)?

(ii) Are the kilonovae produced in NS-NS merger events with $\mathrm{BH}$ remnant different from those produced in merger events with an NS remnant?

(iii) How does the binary system inclination angle influence the kilonova characteristics (colour, peak luminosity)?

Theory predicts that the nature of the progenitor and the merger remnant along with inclination angle of the binary could have an impact on the observable feature of the transient (Roberts et al. 2011). Hydrodynamical simulations show for example that an NS-BH merger is expected to dynamically eject more mass than an NS-NS coalescence (Rosswog 2015), thus generating a more luminous transient. On the other hand, during a BNS merger a part of the ejecta is the result of shocks that emerge from the contact interfaces between the stars. This matter component reaches large enough temperatures $(\sim \mathrm{MeV})$ to undergo fast positron captures and can thus reach electron fractions that substantially differ from the original, very low beta-equilibrium values. The same is true if a massive NS survives at least temporarily the merger event. In this case, strong neutrino-driven winds emerge with a range of electron fractions from $Y_{e} \sim 0.2-0.4$ (Perego et al. 2014). For both types of ejecta - shock- or neutrino-driven - a substantial mass fraction is above the critical value $Y_{e}^{\text {crit }}=0.25$ above which no more lanthanides are produced (Korobkin et al. 2012). Therefore 


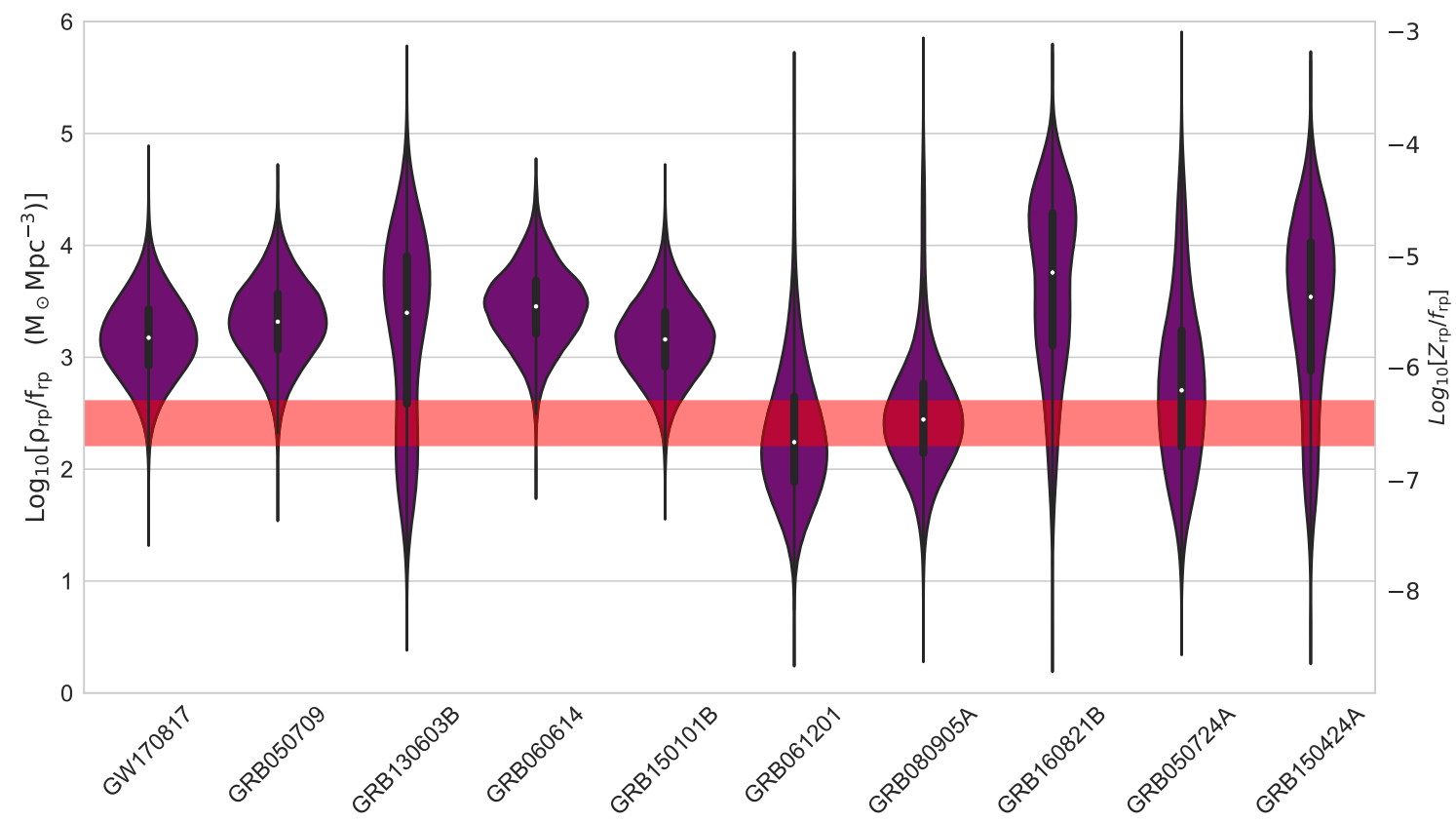

Figure 10. Distributions of average local $r$-process elements density and fraction estimated for the events GW170817, GRB050709, GRB151010B, GRB130603B, GRB060614, GRB061201, GRB080905A, 160821B, GRB050724A, and GRB150424A. The red band represents $r$-process mass fractions obtained from Solar system measurements (Arnould, Goriely \& Takahashi 2007).

the resulting transients are blue. These components, if present, are ejected mainly perpendicularly to the orbital plane inducing in this way a viewing angle dependence (Wanajo et al. 2014; Wollaeger et al. 2018). The angular dependence may reflect in a shift of the kilonova luminosity distribution towards higher magnitudes in the optical bands. This could be verified in the near future, when kilonovae at larger viewing angle will be likely observed in association with GW events. Furthermore, a BNS coalescence could result in the formation of a highly magnetized fast spinning NS, which can be either stable or centrifugally supported by rigid or differential rotation and undergo a delayed collapse into a $\mathrm{BH}$ (Giacomazzo \& Perna 2013; Fryer et al. 2015; Giacomazzo et al. 2015; Ciolfi et al. 2017; Piro, Giacomazzo \& Perna 2017; Radice et al. 2018a). In this scenario the NS dipole spindown emission would constitute an additional source of energy that would heat the ejecta and boost its expansion resulting, once again in different observational features (which would depend also on the remnant NS parameters; Gao et al. 2013; Yu, Zhang \& Gao 2013; Metzger \& Piro 2014; Siegel \& Ciolfi 2016a,b). On the other hand, the presence of the accompaying neutrino-driven wind might prevent the emergence of an sGRB (Murguia-Berthier et al. 2014, 2017a). In this scenario, we could expect the presence of transients with higher luminosities and a spectrum peaked at higher energies not associated with sGRBs, either due to the orientation of the observer or the hampering of the jet. In the case this scenario occurs in a substantial fraction of BNS merger - and considering these sources as proper kilonovae we could expect a shift of the luminosity distribution towards lower magnitudes in (at least) the optical filters.

We are now seeing a renaissance in both the ejecta and light-curve models from kilonova. Improvements in theory are eliminating or placing constraints on uncertainties in the nuclear heating, atomic opacities, and transport methods. In addition, a better understanding of the ejection properties are producing more physical ejecta profiles that will lead to more accurate ties between emission and ejecta masses. With these models, the electromagnetic detections will provide a tight connection to the properties of the mergers. Combined with GW detections, these observations will be able to assess the nature of the progenitor and the merger remnant and measure the viewing angle. These join GW-electromagnetic observations will also place constraints on the role of shocks in the afterglow emission. Assessing whether different remnants lead to different kilonova events is important because, if true, would allow us to identify the nature of the remnant from the only electromagnetic emission, therefore even in case of poor signal to noise ratio of GW post-merger signal.

\section{ACKNOWLEDGEMENTS}

This work was initiated and supported by the 2017 Kavli Summer Program in Astrophysics at the Niels Bohr Institute in Copenhagen, and the authors would like to thank DARK at the University of Copenhagen for incredible hospitality. The 2017 Kavli Summer Program was supported by the Kavli Foundation, Danish National Research Foundation (DNRF), the Niels Bohr International Academy, and DARK

AMB acknowledges support from a UCMEXUS-CONACYT Doctoral Fellowship.

MC is supported by the David and Ellen Lee Postdoctoral Fellowship at the California Institute of Technology.

TD acknowledges support by the European Union's Horizon 2020 research and innovation program under grant agreement No. 749145, BNSmergers.

RJF is supported in part by NASA grant NNG17PX03C, NSF grant AST-1518052, the Gordon \& Betty Moore Foundation, the Heising-Simons Foundation, and by fellowships from the Alfred P. Sloan Foundation and the David and Lucile Packard Foundation.

ER-R is supported in part by David and Lucile Packard Foundation and the Niels Bohr Professorship from the DNRF.

SA acknowledges Giulia Stratta, Daniele Malesani, Andrea Melandri, and Enzo Brocato for useful discussions. 
The authors acknowledge the anonymous referee for his/her suggestions that help us to improve the presentation of our results.

\section{REFERENCES}

Abadie J. et al., 2010, Class. Quantum Gravity, 27, 173001

Abbott B. P. et al., 2017a, Phys. Rev. Lett., 119, 161101

Abbott B. P. et al., 2017b, ApJ, 848, L12

Abbott B. P. et al., 2017c, ApJ, 848, L13

Abbott B. P. et al., 2017d, ApJ, 850, L39

Arnould M., Goriely S., Takahashi K., 2007, Phys. Rep., 450, 97

Barnes J., Kasen D., 2013, ApJ, 775, 18

Barnes J., Kasen D., Wu M.-R., Martínez-Pinedo G., 2016, ApJ, 829, 110

Bauswein A., Baumgarte T. W., Janka H.-T., 2013, Phys. Rev. Lett., 111, 131101

Bauswein A., Just O., Janka H.-T., Stergioulas N., 2017, ApJ, 850, L34

Berger E. et al., 2005, Nature, 438, 988

Berger E., Cenko S. B., Fox D. B., Cucchiara A., 2009, ApJ, 704, 877

Berger E., Fong W., Chornock R., 2013, ApJ, 774, L23

Chornock R. et al., 2017, ApJ, 848, L19

Ciolfi R., Siegel D. M., 2015, ApJ, 798, L36

Ciolfi R., Kastaun W., Giacomazzo B., Endrizzi A., Siegel D. M., Perna R., 2017, Phys. Rev. D, 95, 063016

Côté B. et al., 2018, ApJ, 855, 99

Coughlin M., Dietrich T., Kawaguchi K., Smartt S., Stubbs C., Ujevic M., 2017, ApJ, 849, 12

Coughlin M. W., Dietrich T., Margalit B., Metzger B. D., 2018a, preprint (arXiv e-prints)

Coughlin M. W. et al., 2018b, MNRAS, 480, 3871

Coughlin M. W. et al., 2019, Publications of the Astronomical Society of the Pacific, 131, 998

Coulter D. A. et al., 2017, Science, 358, 1556

Covino S. et al., 2006, A\&A, 447, L5

D’Avanzo P. et al., 2018, A\&A, 613, L1

Dessart L., Ott C. D., Burrows A., Rosswog S., Livne E., 2009, ApJ, 690, 1681

Dietrich T., Ujevic M., 2017, Class. Quantum Gravity., 34, 105014

Dietrich T., Bernuzzi S., Ujevic M., Brügmann B., 2015, Phys. Rev. D, 91, 124041

Dominik M., Belczynski K., Fryer C., Holz D. E., Berti E., Bulik T., Mandel I., O’Shaughnessy R., 2012, ApJ, 759, 52

Drout M. R. et al., 2017, Science, 358, 1570

Eichler D., Livio M., Piran T., Schramm D. N., 1989, Nature, 340, 126

Fernández R., Metzger B. D., 2013, MNRAS, 435, 502

Fong W. et al., 2016, ApJ, 833, 151

Fox D. B. et al., 2005, Nature, 437, 845

Fryer C. L., Woosley S. E., Hartmann D. H., 1999, ApJ, 526, 152

Fryer C. L., Belczynski K., Ramirez-Ruiz E., Rosswog S., Shen G., Steiner A. W., 2015, ApJ, 812, 24

Gao H., Ding X., Wu X.-F., Zhang B., Dai Z.-G., 2013, ApJ, 771, 86

Ghirlanda G. et al., 2019, Science, 363, 968

Giacomazzo B., Perna R., 2013, ApJ, 771, L26

Giacomazzo B., Zrake J., Duffell P. C., MacFadyen A. I., Perna R., 2015, ApJ, 809, 39

Goldstein A. et al., 2017, ApJ, 848, L14

Gompertz B. P. et al., 2018, ApJ, 860, 62

Grossman D., Korobkin O., Rosswog S., Piran T., 2014, MNRAS, 439, 757

Haggard D., Nynka M., Ruan J. J., Kalogera V., Cenko S. B., Evans P., Kennea J. A., 2017, ApJ, 848, L25

Hallinan G. et al., 2017, Science, 358, 1579

Hjorth J. et al., 2005, Nature, 437, 859

Hotokezaka K., Kiuchi K., Kyutoku K., Okawa H., Sekiguchi Y.-. ichiro ., Shibata M., Taniguchi K., 2013, Phys. Rev. D, 87, 024001

Jin Z.-P. et al., 2016, Nat. Commun., 7, 12898

Jin Z.-P. et al., 2018, ApJ, 857, 128
Jin Z.-P., Li X., Cano Z., Covino S., Fan Y.-Z., Wei D.-M., 2015, ApJ, 811, L22

Just O., Bauswein A., Pulpillo R. A., Goriely S., Janka H.-T., 2015, MNRAS, 448,541

Kasen D., Badnell N. R., Barnes J., 2013, ApJ, 774, 25

Kasen D., Fernández R., Metzger B. D., 2015, MNRAS., 450, 1777

Kasen D., Metzger B., Barnes J., Quataert E., Ramirez-Ruiz E., 2017, Nature, 551, 80

Kasliwal M. M. et al., 2017a, Science, 358, 1559

Kasliwal M. M., Korobkin O., Lau R. M., Wollaeger R., Fryer C. L., 2017b, ApJ, 843, L34

Kasliwal M. M., Korobkin O., Lau R. M., Wollaeger R., Fryer C. L., 2017c, ApJ, 843, L34

Kilpatrick C. D. et al., 2017, Science, 358, 1583

Kocevski D. et al., 2010, MNRAS, 404, 963

Korobkin O., Rosswog S., Arcones A., Winteler C., 2012, MNRAS, 426, 1940

Lattimer J. M., Schramm D. N., 1974, ApJ, 192, L145

Lattimer J. M., Schramm D. N., 1976, ApJ, 210, 549

Lazzati D., López-Cámara D., Cantiello M., Morsony B. J., Perna R., Workman J. C., 2017, ApJ, 848, L6

Lee W. H., Ramirez-Ruiz E., 2007, New J. Phys., 9, 17

Lee W. H., Ramirez-Ruiz E., Page D., 2005, ApJ, 632, 421

Lee W. H., Ramirez-Ruiz E., López-Cámara D., 2009, ApJ, 699, L93

Lehner L., Liebling S. L., Palenzuela C., Caballero O. L., O'Connor E., Anderson M., Neilsen D., 2016, Class. Quantum Gravity, 33, 184002

Li L.-X., Paczyński B., 1998, ApJ, 507, L59

Macias P., Ramirez-Ruiz E., 2018, ApJ, 860, 89

Madau P., Dickinson M., 2014, ARA\&A, 52, 415

Malesani D. et al., 2007, A\&A, 473, 77

Margutti R. et al., 2017, ApJ, 848, L20

Matsumoto T., Ioka K., Kisaka S., Nakar E., 2018, ApJ, 861, 55

Matteo T. D., Perna R., Narayan R., 2002, ApJ, 579, 706

Metzger B. D. et al., 2010, MNRAS, 406, 2650

Metzger B. D., 2017, Living Rev. Rel., 20, 3

Metzger B. D., Berger E., 2012, ApJ, 746, 48

Metzger B. D., Fernández R., 2014, MNRAS, 441, 3444

Metzger B. D., Piro A. L., 2014, MNRAS, 439, 3916

Metzger B. D., Piro A. L., Quataert E., 2008, MNRAS, 390, 781

Metzger B. D., Thompson T. A., Quataert E., 2018, ApJ, 856, 101

Mochkovitch R., Hernanz M., Isern J., Martin X., 1993, Nature, 361, 236

Mooley K. P. et al., 2017, Nature, 554, 207

Murguia-Berthier A. et al., 2017a, ApJ, 835, L34

Murguia-Berthier A. et al., 2017b, ApJ, 848, L34

Murguia-Berthier A., Montes G., Ramirez-Ruiz E., De Colle F., Lee W. H., 2014, ApJ, 788, L8

Nagakura H., Hotokezaka K., Sekiguchi Y., Shibata M., Ioka K., 2014, ApJ, 784, L28

Nakar E., 2007, Phys. Rep., 442, 166

Narayan R., Paczynski B., Piran T., 1992, ApJ, 395, L83

Nicuesa Guelbenzu A. et al., 2012, A\&A, 548, A101

O’Shaughnessy R., Belczynski K., Kalogera V., 2008, ApJ, 675, 566

Paczynski B., 1991, Acta Astron., 41, 257

Pannarale F., Ohme F., 2014, ApJ, 791, L7

Perego A., Rosswog S., Cabezón R. M., Korobkin O., Käppeli R., Arcones A., Liebendörfer M., 2014, MNRAS, 443, 3134

Pian E. et al., 2017, Nature, 551, 67

Piran T., Nakar E., Rosswog S., 2013, MNRAS, 430, 2121

Piro A. L., Giacomazzo B., Perna R., 2017, ApJ, 844, L19

Planck Collaboration XIII 2016, A\&A, 594, A13

Radice D., Galeazzi F., Lippuner J., Roberts L. F., Ott C. D., Rezzolla L., 2016, MNRAS, 460, 3255

Radice D., Perego A., Bernuzzi S., Zhang B., 2018a, MNRAS, 481, 3670

Radice D., Perego A., Hotokezaka K., Bernuzzi S., Fromm S. A., Roberts L. F., 2018b, ApJ, 869, 130

Roberts L. F., Kasen D., Lee W. H., Ramirez-Ruiz E., 2011, ApJ, 736, L21 
Rossi A. et al., 2019, preprint (arXiv e-prints)

Rosswog S., 2013, Phil. Trans. R. Soc. A, 371, 20120272

Rosswog S., 2015, Int. J. Mod. Phys. D, 24, 1530012

Rosswog S., Liebendörfer M., Thielemann F.-K., Davies M. B., Benz W., Piran T., 1999, A\&A, 341, 499

Rowlinson A. et al., 2010, MNRAS, 408, 383

Salafia O. S., Colpi M., Branchesi M., Chassande-Mottin E., Ghirlanda G., Ghisellini G., Vergani S. D., 2017, ApJ, 846, 62

Sari R., Piran T., Narayan R., 1998, ApJ, 497, L17

Savchenko V. et al., 2017, ApJ, 848, L15

Shappee B. J. et al., 2017, Science, 358, 1574

Shen S., Cooke R. J., Ramirez-Ruiz E., Madau P., Mayer L., Guedes J., 2015, ApJ, 807, 115

Siegel D. M., Ciolfi R., 2016a, ApJ, 819, 14

Siegel D. M., Ciolfi R., 2016b, ApJ, 819, 15

Siegel D. M., Metzger B. D., 2017, Phys. Rev. Lett., 119, 231102

Siegel D. M., Ciolfi R., Rezzolla L., 2014, ApJ, 785, L6

Smartt S. J. et al., 2017, Nature, 551, 75 EP

Stratta G. et al., 2007, A\&A, 474, 827

Tanaka M., Hotokezaka K., 2013, ApJ, 775, 113
Tanvir N. R. et al., 2015, GRB Coordinates Network, Circular Service, No. 18100, \#1. Available at: http://adsabs.harvard.edu/abs/2015GCN.18100 ....1T

Tanvir N. R. et al., 2017, ApJ, 848, L27

Tanvir N. R., Levan A. J., Fruchter A. S., Hjorth J., Hounsell R. A., Wiersema K., Tunnicliffe R. L., 2013, Nature, 500, 547 EP

Troja E. et al., 2016, ApJ, 827, 102

Troja E. et al., 2017, Nature, 551, 71

Troja E. et al., 2018a, Nat. Commun., 9, 4089

Troja E. et al., 2018b, MNRAS, 478, L18

Wanajo S., Sekiguchi Y., Nishimura N., Kiuchi K., Kyutoku K., Shibata M., 2014, ApJ, 789, L39

Wollaeger R. T. et al., 2018, MNRAS, 478, 3298

Yang B. et al., 2015, Nat. Commun., 6, 7323

Yu Y.-W., Zhang B., Gao H., 2013, ApJ, 776, L40

Zhang B. et al., 2007, ApJ, 655, 989

Zhu Y. et al., 2018, ApJ, 863, L23

\section{APPENDIX A:}

Table A1. Summary of all GRBs in our samples. Ejecta mass and lanthanide fraction are given for the events with a confirmed kilonova detection and for those with informative upper limits are provided. The reported uncertainties correspond to a 90 per cent of confidence interval.

\begin{tabular}{lccccc}
\hline GRB & Ref. & Kilonova & $M_{\mathrm{ej}}\left(\mathrm{M}_{\odot}\right)$ & $X_{\text {lan }}$ & Redshift \\
\hline 170817A (GW170817) & 1 & Yes & $3.87_{-1.44}^{+3.39} \times 10^{-2}$ & $2.71_{-2.03}^{+8.60} \times 10^{-4}$ & 0.0099 \\
130603B & 2,3 & Yes & $7.46_{-7.29}^{+43.97} \times 10^{-2}$ & $5.36_{-5.36}^{+64.63} \times 10^{-3}$ & 0.356 \\
050709 & $4,5,6,7$ & Yes & $5.11_{-2.13}^{+2.98} \times 10^{-2}$ & $4.49_{-4.45}^{+49.60} \times 10^{-5}$ & 0.161 \\
060614 & $8,9,10$ & Yes & $7.73_{-2.85}^{+1.90} \times 10^{-2}$ & $2.24_{-2.23}^{+36.73} \times 10^{-6}$ & 0.125 \\
$150101 \mathrm{~B}$ & 11,12 & Recently claimed by 12 & $3.71_{-1.56}^{+3.12} \times 10^{-2}$ & $4.19_{-4.16}^{+889.60} \times 10^{-7}$ & 0.134 \\
140903A & 13 & No & - & - & 0.351 \\
050724A & 14,15 & Recently claimed by 24 & $1.24_{-1.09}^{+39.99} \times 10^{-2}$ & $0.09_{-0.09}^{+22.56} \times 10^{-4}$ & 0.257 \\
061201 & 16 & Recently claimed by 24 & $4.20_{-2.91}^{+38.34} \times 10^{-3}$ & $0.07_{-0.07}^{+361.50} \times 10^{-4}$ & $0.111^{a}$ \\
080905A & 17,18 & Recently claimed by 24 & $6.98_{-4.58}^{+44.01} \times 10^{-3}$ & $1.41_{-1.41}^{+200.38} \times 10^{-4}$ & 0.1218 \\
070724A & 19,20 & No & - & - & 0.457 \\
160821B & 21,22 & Recently claimed by 24 & $1.74_{-1.69}^{+6.97} \times 10^{-1}$ & $1.87_{-1.87}^{+175.29} \times 10^{-4}$ & 0.16 \\
150424A & 22,23 & Recently claimed by 24 & $9.66_{-9.45}^{+56.04} \times 10^{-2}$ & $0.15_{-0.15}^{+188.15} \times 10^{-4}$ & 0.30 \\
\hline
\end{tabular}

Note: ${ }^{a}$ This event has been associated to a galaxy at the redshift reported here or to the cluster Abell 995 at $z=0.084$. Gompertz et al. (2018) employed for this event the latter value, while we choose the former in order to be more conservative.

References: (1) Abbott et al. (2017b), (2) Tanvir et al. (2013), (3) Berger et al. (2013), (4) Fox et al. (2005), (5) Hjorth et al. (2005), (6) Covino et al. (2006), (7) Jin et al. (2016), (8) Zhang et al. (2007), (9) Jin et al. (2015), (10) Yang et al. (2015), (11) Fong et al. (2016), (12) Troja et al. (2018a), (13) Troja et al. (2016), (14) Berger et al. (2005), (15) Malesani et al. (2007) (16) Stratta et al. (2007), (17) Nicuesa Guelbenzu et al. (2012), (18) Rowlinson et al. (2010), (19) Berger et al. (2009), (20) Kocevski et al. (2010), (21) Kasliwal et al. (2017c), (22) Jin et al. (2018), (23) Tanvir et al. (2015), (24) Rossi et al. (2019). 

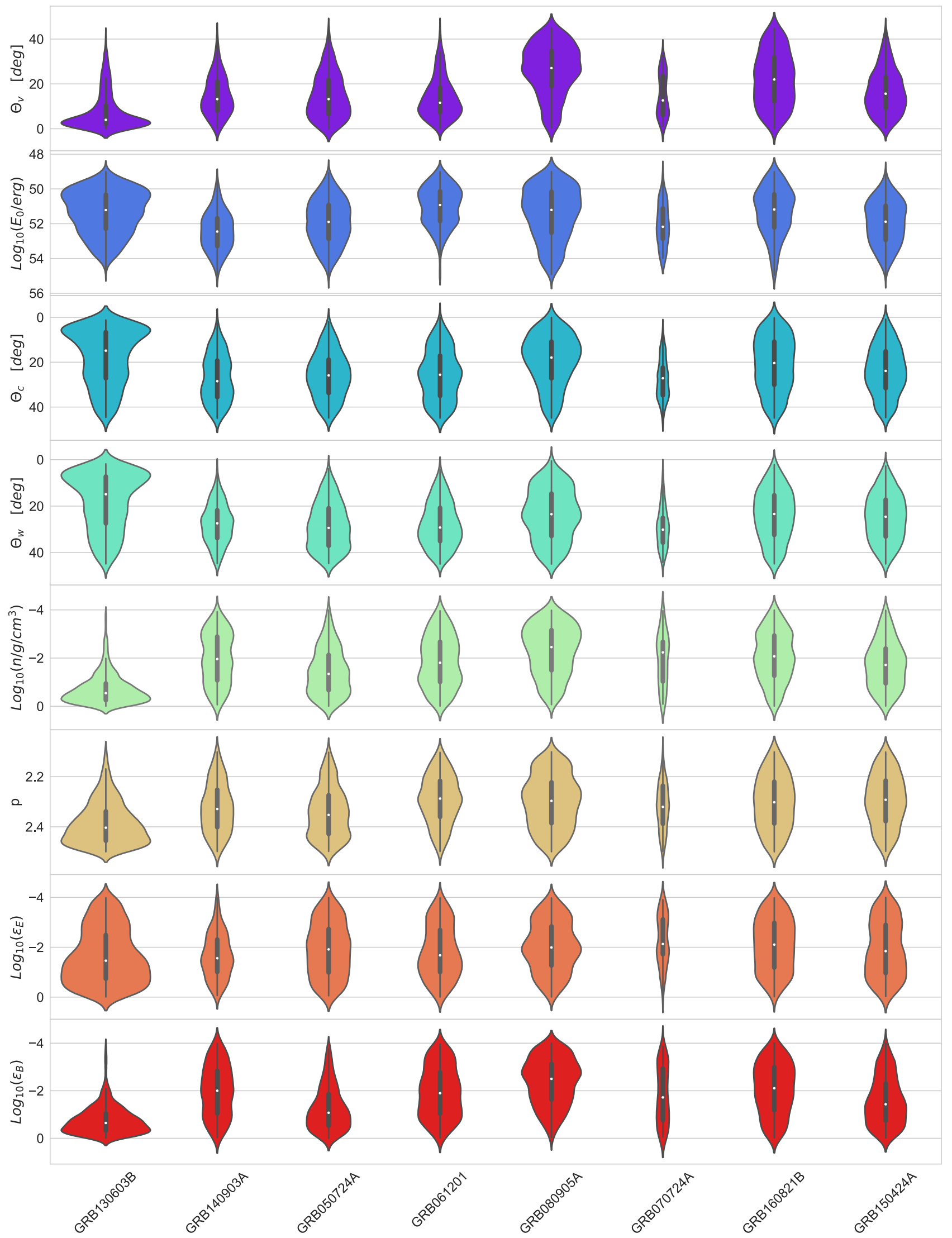

Figure A1. Distributions of the afterglow parameters obtained for the events fitted by the afterglow+kilonova models. 

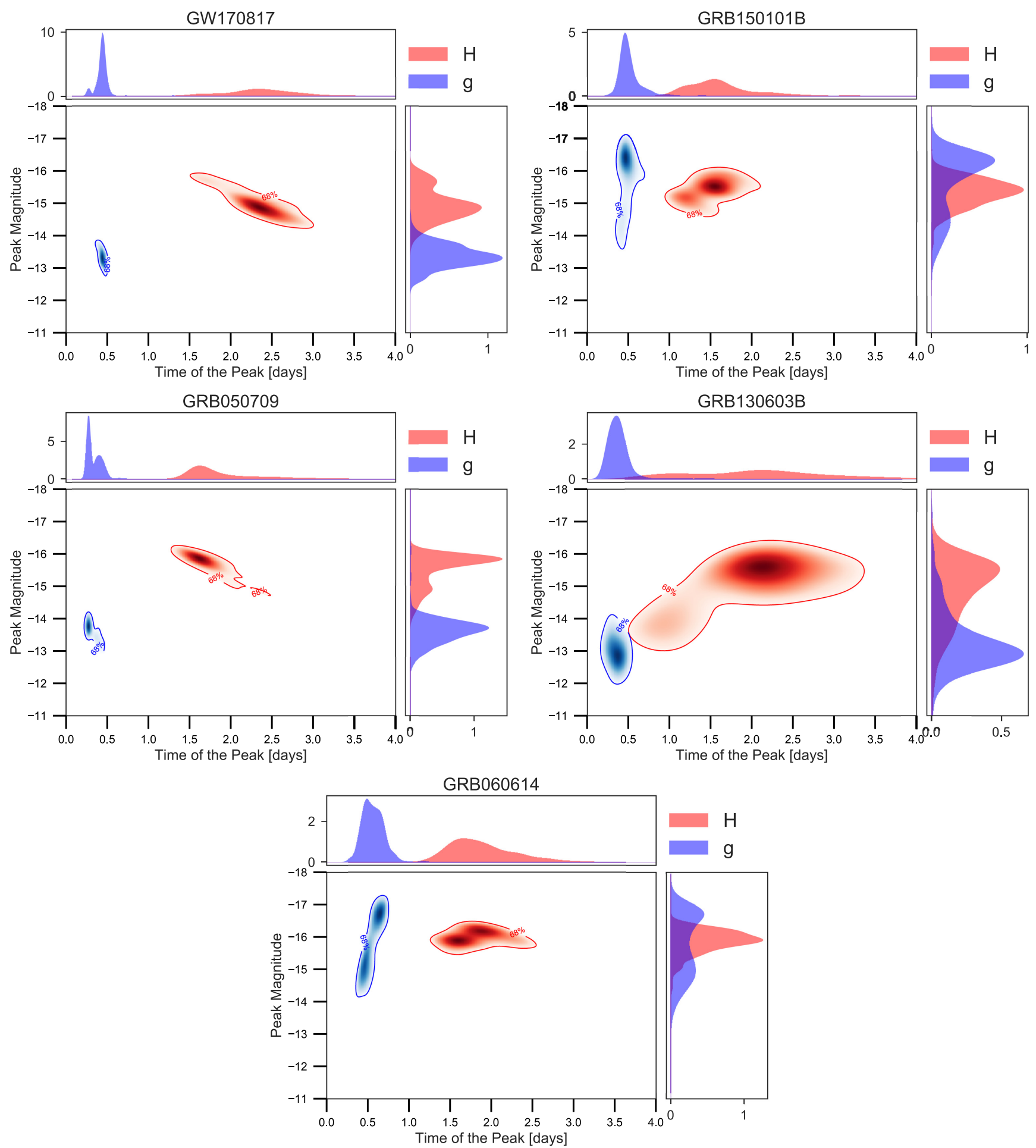

Figure A2. Probability density distribution (within 68 per cent of confidence) in a peak time-peak magnitude plane in $g$ (blue) and $H$ (red) filters for the events GW170817, GRB150101B, GRB050709, GRB130603B, and GRB060614. Top and right-hand panels show the marginalized distributions in peak magnitude and peak time, respectively. 

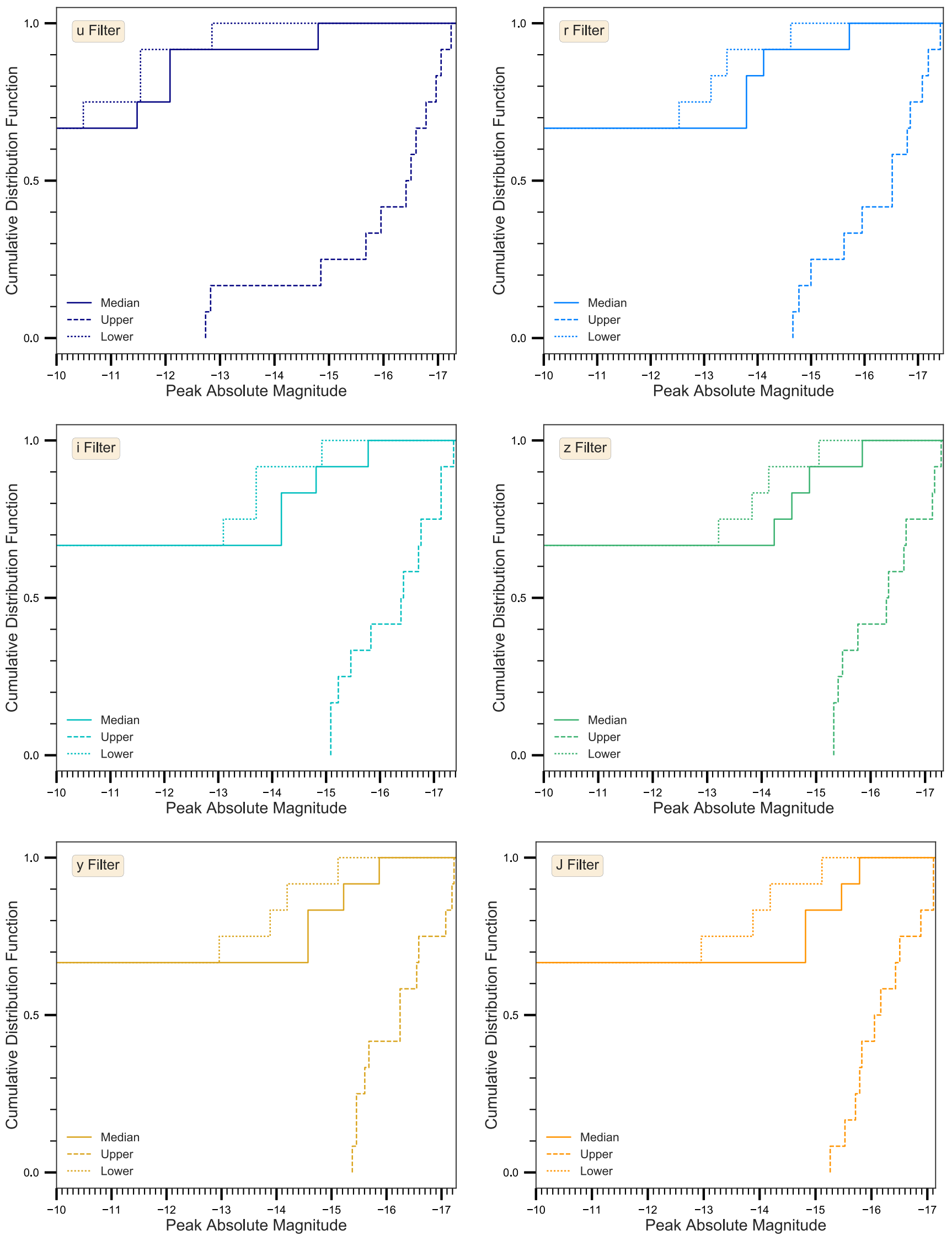

Figure A3. Luminosity distributions in $u, r, i, z, y, J$, and $K$ filters. 


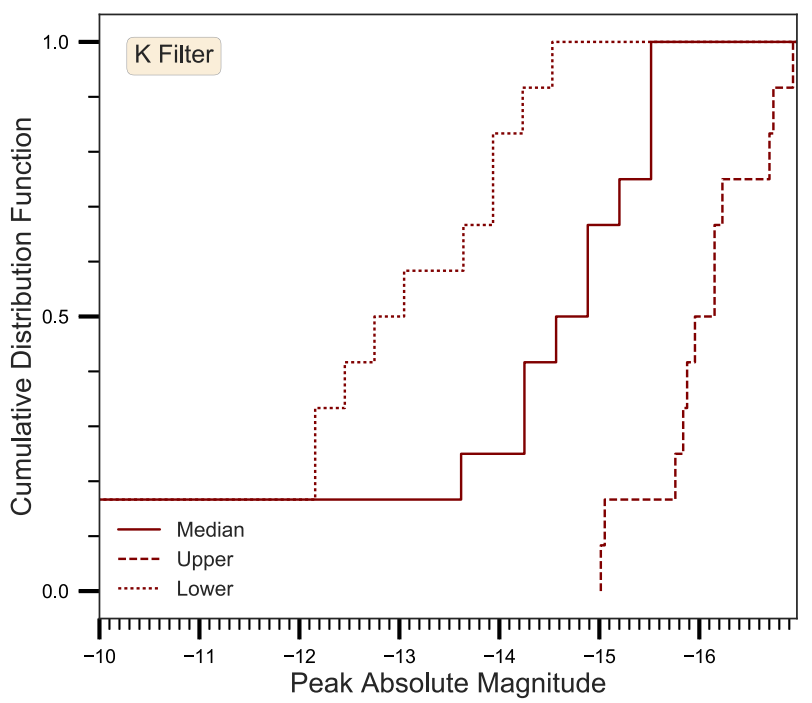

Figure A3 - Continued 

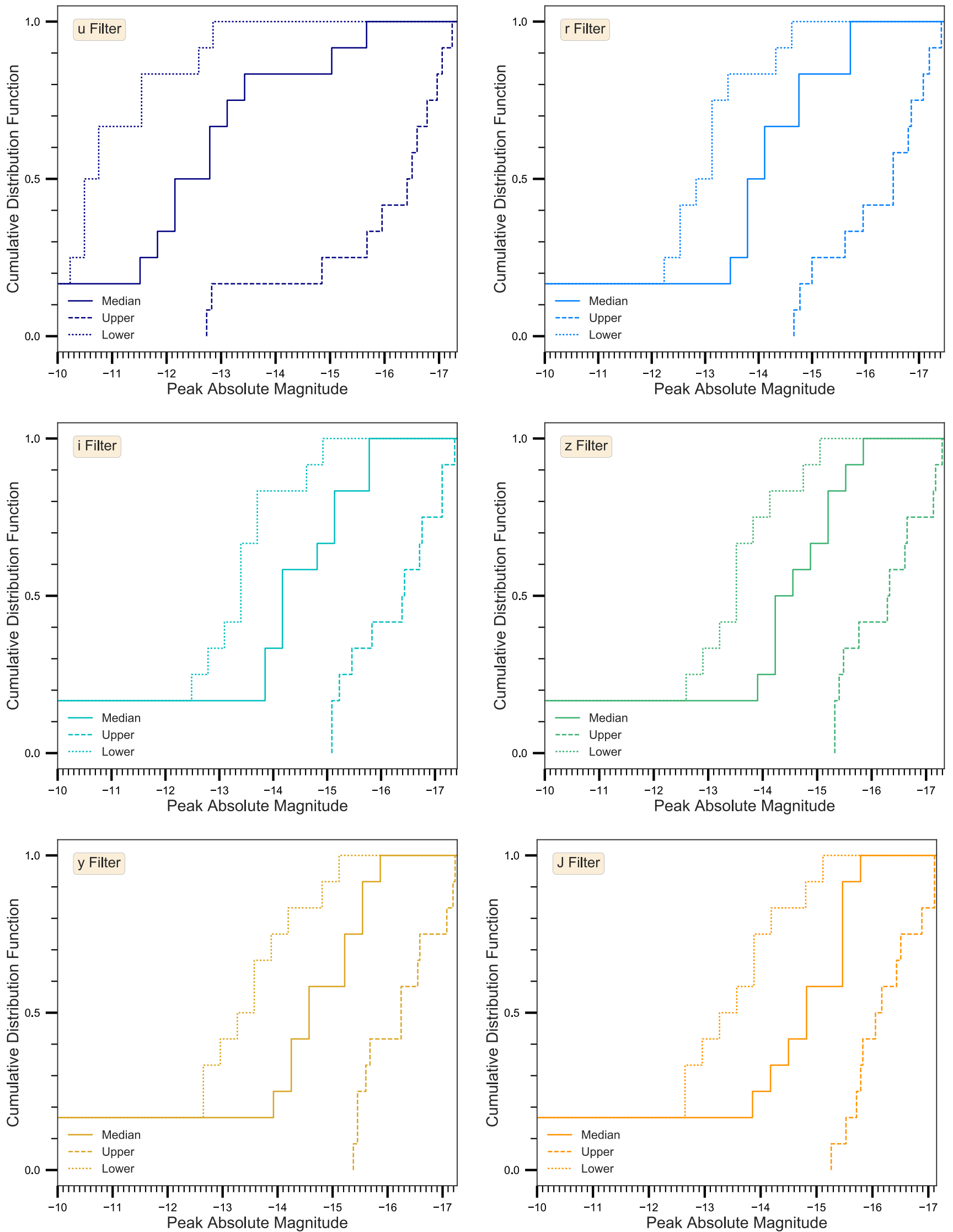

Figure A4. Same as Fig. A3 but here we promoted GRB150101B, GRB050724A, GRB061201, GRB080905A, GRB150424A, and GRB160821B to kilonova events. 


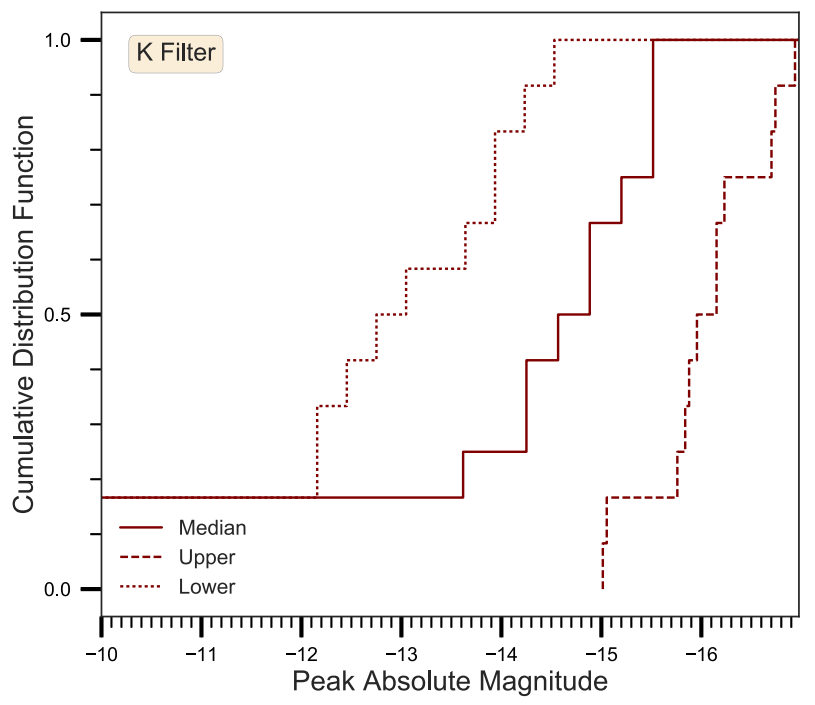

Figure A4 - Continued

This paper has been typeset from a $\mathrm{T}_{\mathrm{E}} \mathrm{X} / \mathrm{L} \mathrm{T}_{\mathrm{E}} \mathrm{X}$ file prepared by the author. 